\title{
Inequalities and Many Phase Transitions in Ferromagnetic Systems
}

\author{
R. Fernández ${ }^{1}$ and J. Slawny ${ }^{2}$ \\ ${ }^{1}$ Department of Mathematics, University of Texas at Austin, Austin, TX 78731, USA \\ ${ }^{2}$ Center for Transport Theory and Mathematical Physics, Virginia Polytechnic Institute and \\ State University, Blacksburg, VA 24061, USA
}

\begin{abstract}
A general construction of ferromagnetic systems with many phase transitions is given. It is based on two new results: an extension of one of the GKS inequalities to not necessarily ferromagnetic interactions, and a uniqueness of the Gibbs state theorem for perturbations of some simple systems at all temperatures.
\end{abstract}

\section{Introduction}

This work arose from an attempt to understand the global structure of phase diagrams of classical ferromagnetic lattice systems. Several techniques have been developed to study the low temperature phase diagrams; among them the Peierls argument $[27,19,6]$, reflection-positivity techniques [14-16], Pirogov-Sinai theory $[29,34,32]$ and its extensions $[3,9,25,34,4,5]$, and algebraic methods peculiar to ferromagnetic systems $[21,23,28,32,26]$. However, very little is known about how this phase diagram changes with the temperature, especially in systems with multispin interactions. Here we explore a general mechanism explaining occurrence of many phase transitions in some ferromagnetic systems as the temperature is varied. In particular we construct models for which the existence of several phase transitions can be proved. Later, in this introduction and in Sect. 5.4, we comment on the relation of this work to an earlier related work by Pfister [28].

The systems considered here are ferromagnetic in the sense that the configuration space is an (abelian) group and the Hamiltonians $H$ are negative definite, i.e. they are linear combinations of characters with negative coefficients. Such systems have a distinguished Gibbs state \langle\rangle$^{+}$which breaks the symmetry of the Hamiltonian in a maximal way. Let $\mathscr{A}_{\beta}(H)$ denote the set of characters $\sigma$ for which $\langle\sigma\rangle_{\beta H}^{+} \neq 0$, where $\beta$ is the inverse temperature. By a general argument, $[30,28], \mathscr{A}_{\beta}$ is becoming larger as $\beta$ increases, it always contains the group $\overline{\mathscr{B}}(H)$ generated by the characters appearing in $H$ and it stabilizes both at low and high temperatures, to $\mathscr{A}_{\infty}(H)$ and to $\mathscr{A}_{0}(H)(=\overline{\mathscr{B}}(H))$ respectively. If $\mathscr{A}_{\infty}(H)$ is strictly larger than $\mathscr{A}_{0}(H)$ one has a phase transition as the temperature is varied. The problem is to investigate at how many temperatures $\mathscr{A}_{\beta}(H)$ is changing. 
Now, one can think about many chains $\ldots \mathscr{C A}_{k-1} \subset \mathscr{A}_{k} \subset \mathscr{A}_{k+1} \subset \ldots$ of translation invariant groups. For instance, one could consider splitting the Hamiltonian $H$ into a sum $\sum H_{k}$ of smaller and smaller summands so that when the temperature is lowered $\mathscr{A}$ is enlarged by adding to it first the characters which are the order parameters of the largest summand, then changed again when the order parameters of the second largest summand develop non-zero expectation values, and so on. In this paper one has the finite abelian group $\mathbf{Z}_{\mathrm{p}}$ at each lattice site (" $\mathbf{Z}_{\mathrm{p}}$-models"). In the examples of [28] $p$ is composite, the interaction is between nearest neighbors and $\mathscr{A}_{k+1}=2 \mathscr{A}_{k}$; in the cases considered here $p$ is prime and $\mathscr{A}_{k+1}$ is obtained from $\mathscr{A}_{k}$ by adding to it the generator $D_{k}$ of $\mathscr{A}_{\infty}\left(H_{k}\right)$ (see the theorem of Sect. 3.2). The Hamiltonians $H_{k}$ are quite arbitrary, apart from a requirement that $D_{k}$ has to be of a regular shape, in a sense. The general case should be some "mixture" of the situation considered here and in [28], but this has not been worked out (see the comments at the end of the paper, where we also show how Pfister's examples fit into our framework).

To explain our general result we start with a discussion of a simple model with several phase transitions. This model will be referred to in the sequel as the "benchmark model." It is defined on a simple square lattice $\mathbf{Z}^{2}$, has spin $\frac{1}{2}$ and Hamiltonian with nearest neighbor vertical bonds, next nearest neighbor horizontal bonds and four-point "plaquette" bonds:

$$
H=-\sum_{x}\left\{a \sigma_{x} \sigma_{x+e^{2}}+b \sigma_{x} \sigma_{x+2 e^{1}}+c \sigma_{x} \sigma_{x+e^{1}} \sigma_{x+e^{2}} \sigma_{x+e^{1}+e^{2}}\right\},
$$

where the sum is over all points $x$ of the lattice, and $\sigma_{x}$ is the usual spin- $\frac{1}{2}$ variable at the point $x$. The same Hamiltonian is also written as

$$
H=-a \sum_{x} \sigma_{A+x}-b \sum_{x} \sigma_{B+x}-c \sum_{x} \sigma_{C+x} \quad a, b, c \geqq 0,
$$

where $A=\left\{0, e^{2}\right\}, B=\left\{0,2 e^{1}\right\}, C=\left\{0, e^{1}, e^{2}, e^{1}+e^{2}\right\} ; A+x$ denotes the translate of $A$ by $x \in \mathbf{Z}^{2}$, and

$$
\sigma_{A}=\prod_{x \in A} \sigma_{x}
$$

We discuss now the number of phase transitions in this system as the temperature is varied.

If $c=0$ and $a$ and $b$ are strictly positive one has two mutually non-interacting Ising models. Therefore the magnetization $\left\langle\sigma_{0}\right\rangle^{+}$is non-zero at temperatures low enough. By the GKS inequalities this is also true for positive $c$.

Let now $a=0$ and let $\tau_{x}=\sigma_{x} \sigma_{x+e^{1}}\left(=\sigma_{D+x}\right.$, where $\left.D=\left\{0, e^{1}\right\}\right)$. Then

$$
H=-b \sum_{x} \tau_{x} \tau_{x+e^{1}}-c \sum_{x} \tau_{x} \tau_{x+e^{2}}
$$

i.e. in terms of the $\tau$-variables one has an Ising model. This suggests that at low enough temperatures, or large $\beta$, and $a=0$

$$
\left\langle\sigma_{D}\right\rangle_{\beta H}^{+} \neq 0 \text {, }
$$

while this expectation value is zero at temperature high enough. That this is indeed the case follows from [23] (see the next section). Moreover, by [23] expectation values in the Gibbs states of (1.2) of $\sigma_{A}$ which are not products of a finite number of 
$\tau_{x}$ are zero at all temperatures. In particular one obtains that for $a=0$,

$$
\left\langle\sigma_{0}\right\rangle_{\beta H}^{+}=0 \text { for any } \beta \neq 0 ;
$$

we want to show that (1.4) holds also for small $a$ for some $\beta$ for which (1.3) holds. Note that a proof of this may require a somewhat subtle argument for one needs a kind of a stability result for Hamiltonians for which the Gibbs state is non-unique [see (1.3)]. Such a result must depend on the observable one considers: with $\sigma_{x} \sigma_{y}$ in place of $\sigma_{x}$ one has that $\left\langle\sigma_{x} \sigma_{y}\right\rangle_{\beta H}^{+} \neq 0$ as soon as $a \neq 0$ (by the Griffiths-KellySherman (GKS) inequalities $[20,24])$.

In conclusion, for $a, b, c>0$ we have that at low temperatures $\left.\left\langle\sigma_{0}\right\rangle^{+}\right\rangle 0$ and $\left\langle\sigma_{D}\right\rangle^{+}>0$, while at high temperature both expectations are zero. The question we focus on is whether both order parameters become non-zero at the same time or, on the contrary, there is an intermediate range of temperatures for which $\left\langle\sigma_{0}\right\rangle^{+}=0$ and $\left\langle\sigma_{D}\right\rangle^{+}>0$ (by GKS, if the magnetization is non-zero then $\left\langle\sigma_{D}\right\rangle^{+}>0$ too).

We prove below that if $a$ is non-zero but small compared with $b$ and $c$ then the latter possibility holds, and there are two phase transitions ocurring at different inverse temperatures $\beta_{1}>\beta_{2}$, where

$$
\beta_{2}=\inf \left\{\beta:\left\langle\sigma_{D}\right\rangle_{\beta H}^{+}>0\right\}
$$

and

$$
\beta_{1}=\inf \left\{\beta:\left\langle\sigma_{0}\right\rangle_{\beta H}^{+}>0\right\} .
$$

Monte Carlo calculations [13] seem to indicate that for $a$ large the two temperatures coincide.

The proof has two ingredients. First, we establish a correlation inequality to majorize the (magnetic) correlation functions of the \langle\rangle$^{+}$-state of (1.1) by the corresponding expectation values in the \langle\rangle$^{+}$-state of the Hamiltonian

$$
-a \sum_{x} \sigma_{A+x}-f(b, c) \sum_{x} \sigma_{D+x},
$$

where $f$ is an increasing function of $b$ and $c$ (Sect. 3.3). If $a=0$ the Hamiltonian (1.1) has a unique Gibbs state which is easily computed and for which the magnetization is zero. By the above mentioned inequality (or by [23]), this implies that the magnetization is also zero for the system with Hamiltonian (1.1) if $a=0$. However, in order to prove the existence of two phase transitions for the interaction (1.1) we need to show that the magnetization of (1.1) is zero for small but non-zero values of $a$.

This follows from our second general result, which is deduced from a general uniqueness criterion of Dobrushin and Shlosman [8]. It states that small perturbations of a Hamiltonian of the form $J \sum_{x} \sigma_{D+x}$ with $D$ of a regular shape (a factorizable trivial system of Sect. 3.2), but otherwise arbitrary, have a unique Gibbs state. We note that this is a uniqueness result which is valid at all temperatures, not only in the high- or the low-temperature region.

(In the present example, (1.7) is the Hamiltonian of the Ising model, and the vanishing of the magnetization for small values of $a$ can be deduced either from the well known explicit formula for the magnetization of the two-dimensional Ising 
model, or a result of Fisher [12]. The later approach works also in higher dimensions. Neither of these methods generalize to our more general setting: the result of [12] depends on a self-avoiding walk representation of the system and on some additional properties characteristic for systems with two-body interactions.)

The paper is organized as follows. After introducing the notation and reviewing some well known results (Sect. 2), we state the two new "ingredients": the inequality and the uniqueness theorem (Sect. 3.1); we deal first with the spin- $-\frac{1}{2}$ case. This allows us to describe the construction of models with many phase transitions as soon as possible (Sect. 3.2), leaving the rest of the paper for the details of the proofs and for extensions of the results. The proof of the inequality is the subject of Sect. 3.3, and we devote Sect. 4 to the proof of the uniqueness theorem. In Sects. 5.1-5.3 we sketch how the results can be extended to the situation when one has at each lattice site $\mathbf{Z}_{\mathrm{p}}$, with $p$ prime, instead of $\mathbf{Z}_{2}$, and in Sect. 5.4 we compare our work with Pfister's. In the appendix we collect some results whose proofs have a stronger algebraic flavour.

\section{The Framework}

The lattice $\mathbf{L}$ is a discrete $\mathbf{Z}^{v}$-invariant subset of $\mathbf{R}^{v}$; in most of the paper we will deal with the case of a simple lattice, i.e. $\mathbf{L}=\mathbf{Z}^{v}$. In the spin- $\frac{1}{2}$ case, which is considered first, the configuration space of the system is

$$
\mathbf{X}=\{-1,1\}^{\mathbf{L}},
$$

which is an abelian group with pointwise multiplication

$$
(X \cdot Y)_{a}=X_{a} Y_{a}
$$

a topological space with the product topology; and a measure space with the $\sigma$-algebra generated by the cylindrical sets. If $\Lambda \subset \mathbf{L}$, we shall denote $\mathbf{X}_{A}$ the set of configurations on $\Lambda: \mathbf{X}_{\Lambda}=\{-1,1\}^{A}$, and for $X \in \mathbf{X}$ its restriction to $A$ is denoted by $X_{\Lambda}$. The configuration equal 1 everywhere will be denoted 1 .

We will consider systems with translation-invariant finite-range interactions. For such system the Hamiltonian is uniquely written in the form

$$
H=-\sum_{B \in \mathscr{B}} J(B) \sigma_{B}
$$

where $\mathscr{B}=\mathscr{B}(H)$ is a $\mathbf{Z}^{v}$-invariant family of finite subsets of $\mathbf{L}$ (the "bonds"), and $J(B)=0$ if diameter of $B$ exceeds certain value.

The function $\sigma_{B}$ is a character of $\mathbf{X}$; moreover

$$
\sigma_{B} \sigma_{C}=\sigma_{B+C},
$$

where $B+C=(B \cup D) \backslash(B \cap D)$ is the symmetric difference. Hence, the group structure of the dual group to $\mathbf{X}$ is isomorphic to the group $\mathscr{P}_{f}(\mathbf{L})$ of finite subsets of $\mathbf{L}$ with the symmetric difference as the group operation. We will consider similarly defined group structure in the set of all subsets of any set.

For a finite set $A \subset \mathbf{L}$, a configuration $Y$ on its complement defines a boundary condition. More generally, we shall consider boundary conditions in which some of the spins of the boundary have been removed. Hence, every partial configuration 
$Y \in \mathbf{X}_{M}$ with $M \subset A^{c}$, defines a boundary condition with the spins on $M$ set in the configuration $Y$, and those outside of $M$ removed. The Hamiltonian on $\Lambda$ for a boundary condition $Y \in \mathbf{X}_{M}, M \subset A^{c}$, is the function

$$
X \mapsto H_{\Lambda}(X \mid Y)=\sum_{\substack{B \subset A \cup M \\ B \cap A \neq \emptyset}} J(B) \sigma_{B \cap M}(Y) \sigma_{B \cap A}(X)
$$

defined for $X \in \mathbf{X}_{\Lambda}$. The Gibbs state in $\Lambda$ for the boundary condition $Y \in \mathbf{X}_{M}$, Hamiltonian $H$ and inverse temperature $\beta$ is the probability measure $\varrho_{A}(\cdot \mid Y)$ on $\mathbf{X}_{\Lambda}$ such that

$$
\varrho_{\Lambda}(X \mid Y)=\frac{e^{-\beta H_{\Lambda}(X \mid Y)}}{Z(\Lambda \mid Y)},
$$

where $Z(\Lambda \mid Y)$ is the normalization factor and $\beta$ is the inverse temperature. The (infinite volume) Gibbs states are the probability measures on $\mathbf{X}$ whose conditional probabilities on $\mathbf{X}_{\Lambda}$ given a configuration $Y \in \mathbf{X}_{\Lambda^{c}}$ are precisely the measures $\varrho_{\Lambda}(\cdot \mid Y)$. The set of such Gibbs states will be denoted as $\Delta(\beta H)$. We shall denote by \langle\rangle$^{+}$, or $\varrho^{+}$, the Gibbs state obtained as the vague limit of the net $\left\{\varrho_{A}(\cdot \mid 1)\right\}$ as $\Lambda$ tends to $\mathbf{L}$ through the family of all finite subsets of $\mathbf{L}$ directed by inclusion. This Gibbs state is translation-invariant.

If the system has a unique Gibbs state - as is the case at high temperatures then this state coincides with $\varrho_{\beta H}^{+}$and it has the property that all the correlation functions $\varrho_{B H}^{+}\left(\sigma_{A}\right)$ vanish unless $\sigma_{A}$ is a product of characters $\sigma_{B}$ with $B \in \mathscr{B}$. Equivalently, we have that if there is a unique Gibbs state then

$$
\mathscr{A}_{\beta}(H)=\left\{A \in \mathscr{P}_{f}(\mathbf{L}): \varrho_{\beta H}^{+}\left(\sigma_{A}\right) \neq 0\right\}
$$

satisfies

$$
\mathscr{A}_{\beta}=\overline{\mathscr{B}},
$$

where $\overline{\mathscr{B}}=\overline{\mathscr{B}}(H)$ is the subgroup of $\mathscr{P}_{f}(\mathbf{L})$ generated by $\mathscr{B}$.

In general $\mathscr{A}_{\beta}$ is a subgroup of $\mathscr{P}_{f}(\mathbf{L})$ which by the GKS inequalities is becoming larger as $\beta$ increases. It contains always $\mathscr{A}_{0}=\overline{\mathscr{B}}(H)$, to which it is equal at sufficiently high temperatures. Elements of $\mathscr{A}_{\beta}$ which are not in $\overline{\mathscr{B}}(H)$ can be considered as order parameters since they tell which part of the (internal) symmetry group of the system is broken (see [33, 30, 21, 28], and [32] for a review). We are interested here in the problem of how $\mathscr{A}_{\beta}$ is changing with the temperature.

By a general argument [23], $\mathscr{A}_{\beta}$ stabilizes at low temperatures, i.e. there is an inverse temperature, say $\beta_{\infty}$, such that $\mathscr{A}_{\beta}$ is the same, say $\mathscr{A}_{\infty}$, for all $\beta>\beta_{\infty} . \mathscr{A}_{\beta}(H)$ can be determined from $\overline{\mathscr{B}}(H)$ in a purely algebraic way $[23,32]$. For simple lattices $\mathscr{A}_{\infty}(H)$ is always generated by translations of a (unique, up to translations) element $D\left(D=\left\{0, e^{1}\right\}\right.$ for the "benchmark model" (1.1) with $\left.a=0\right)$. To compute $D$, and to prove its existence, one introduces in $\mathscr{P}_{f}\left(\mathbf{Z}^{v}\right)$ the structure of a ring with product

$$
C \cdot D \equiv \sum_{a \in C} D+a
$$

This allows the identification of $\mathscr{P}_{f}(\mathbf{L})$ with the group algebra $\mathbf{Z}_{2}\left[\mathbf{Z}^{v}\right]$ of $\mathbf{Z}^{v}$ with coefficients in $\mathbf{Z}_{2} . \mathbf{Z}_{2}\left[\mathbf{Z}^{v}\right]$ is a unique factorization domain, and $D$ is the greatest common divisor of $\mathscr{B}$ (see $[23,32])$ :

$$
D=\text { g.c.d. }(\mathscr{B}) \text {. }
$$


In particular

$$
\varrho^{+}\left(\sigma_{D}\right)>0
$$

at low enough temperatures. (In case of general lattices $\overline{\mathscr{B}}(H)$ is a $\mathbf{Z}_{2}\left[\mathbf{Z}^{v}\right]$-module, and $\mathscr{A}_{\infty}$ is identified with the second dual of this module, [31]). Generalization of this structure to the $\mathbf{Z}_{n}$-case is described in Sect. 5 .

Let us finally recall the high temperature expansion (HTE) [33, 21]. Given a Hamiltonian $H$ on a finite subset $A$ of $\mathbf{L}$ :

$$
H=-\sum_{C \in \mathscr{C}} J(C) \sigma_{C},
$$

where $\mathscr{C}$ is some family of subsets of $A$ [and $J(C)$ need not be positive], the partition function

$$
Z(\Lambda ; H)=\sum_{X \in \mathbf{X}_{\Lambda}} \exp -H(X)
$$

is written as follows:

$$
Z(\Lambda ; H)=2^{|\Lambda|}\left(\prod_{C \in \mathscr{C}} \cosh J(C)\right) Z^{0}(\Lambda ; H) .
$$

The non-trivial part in this expression is the "reduced partition function"

$$
Z^{0}(\Lambda ; H)=\sum_{\alpha \in \mathscr{K}(\mathscr{C})} \prod_{C \in \alpha} \tanh J(C)
$$

where $\mathscr{K}(\mathscr{C})$ denotes the set of cycles associated with the family $\mathscr{C}$ of bonds:

$$
\mathscr{K}(\mathscr{C})=\left\{\text { families } \alpha \subset \mathscr{C} \mid \sum_{C \in \alpha} C=0\right\} .
$$

We note that if $\mathscr{K}(\mathscr{C}) \neq\{0\}$, then the decomposition of each set $A \in \mathscr{C}$ into a sum of bonds of $\mathscr{C}$ is not unique. Indeed, if $\alpha_{A}$ is a family of sets in $\mathscr{C}$ with sum $A$, then for each $\alpha \in \mathscr{K}(\mathscr{C})$ the family $\alpha+\alpha_{A}$ (where " + " is the symmetric difference of sets) also adds up to $A$. The HTE of the correlation functions takes the form

$$
\left\langle\sigma_{A}\right\rangle_{(\Lambda ; H)}= \begin{cases}0 & \text { if } A \notin \overline{\mathscr{C}} \\ {\left[Z^{0}(\Lambda ; H)\right]^{-1} \sum_{\alpha \in \mathscr{K}(\mathscr{C})} \prod_{C \in \alpha+\alpha_{A}} \tanh J(C)} & \text { if } A \in \overline{\mathscr{C}},\end{cases}
$$

where in the second line $\alpha_{A}$ is any fixed family of bonds whose symmetric difference is $A$.

\section{3. $\operatorname{Spin} \frac{1}{2}$}

For the ease of the exposition we deal first with the spin- $\frac{1}{2}$ case. Extension of these results is given in Sect. 5 .

\subsection{Two New Results: An Inequality and a Uniqueness Theorem}

The first new result which will be used in our discussion of the examples of models with several phase transitions is the following inequality, which will be proved in Sect. 3.3. 
Theorem 3.1. Consider two ferromagnetic translation invariant finite range interactions:

$$
H_{1}=-\sum_{B \in \mathscr{B}_{1}} J_{1}(B) \sigma_{B}, \quad H_{2}=-\sum_{B \in \mathscr{B}_{2}} J_{2}(B) \sigma_{B}
$$

such that each bond of the first interaction is a symmetric difference of a number of bonds of the second one, i.e.,

$$
\mathscr{B}_{1} \subset \overline{\mathscr{B}}_{2} \text {. }
$$

Then there exists a smooth, strictly increasing function $\beta \mapsto f(\beta)$ with the properties

$$
f(\beta) \searrow 0 \quad \text { as } \beta \searrow 0, \quad f(\beta) \nearrow \infty \quad \text { as } \beta \nearrow \infty,
$$

and such that

$$
\varrho_{\beta H_{1}+K}^{+}\left(\sigma_{A}\right) \leqq \varrho_{f(\beta) H_{2}+K}^{+}\left(\sigma_{A}\right)
$$

for every ferromagnetic interaction $K$ (not necessarily of a finite range), and every finite $A \subset \mathbf{L}$.

For the second result the Hamiltonian $\mathrm{H}_{2}$ will be of a very simple form: We will call a system trivial if its fundamental family of bonds $\mathscr{B}_{0}$ consists of exactly one bond, i.e.

$$
\mathscr{B}=\left\{C+a: a \in \mathbf{Z}^{\nu}\right\}
$$

for some finite $C \subset \mathbf{Z}^{v}$ called the fundamental bond; and

$$
H=-J \sum_{x} \sigma_{C+x}
$$

A system is factorizable if all its bonds are cartesian products of one-dimensional bonds. In particular, a fundamental bond of a factorizable trivial system is of the form

$$
C=C_{1} \times \ldots \times C_{v}
$$

where each $C_{i}$ is a subset of the $i^{\text {th }}$ coordinate axis of $\mathbf{Z}^{v}$. Note that this is equivalent to

$$
C=C_{1} \cdot \ldots \cdot C_{v},
$$

with "." defined in (2.6). We note that a product of two sets is factorizable if and only if each of the sets is (the "only if" part follows from the fact that $\mathbf{Z}_{2}\left[\mathbf{Z}^{\nu}\right]$ is a unique factorization domain).

Factorizable trivial systems are simple to analyze and they have a unique Gibbs state at all temperatures. However, we will need the following stronger result.

Theorem 3.2. Consider a trivial interaction $H=-J \sum_{x} \sigma_{C+x}$ with a factorizable $C$. Then for any positive $r$ there exists $R_{r, C, J}>0$ such that for any perturbation $K=\sum K(B) \sigma_{B}$ with range less than $r$ and

$$
\sup _{B}|K(B)|<R_{r, C, J}
$$


the Hamiltonian $H+K$ has a unique Gibbs state, i.e. $\Delta(H+K)$ has exactly one element.

[Note that we are absorbing the inverse temperature $\beta$ in the couplings $J$ and $K(B)$.] This will be proved in Sect. 4 . We have no doubt that the theorem, thought not the proof of Sect. 4.3, holds without the assumption that $C$ is factorizable.

In our construction of models with many phase transitions we will use only the following corollary of Theorem 3.1 and 3.2. If $\mathscr{B}_{1}, \ldots, \mathscr{B}_{k}$ are (families of) subsets of the lattice then $\left[\mathscr{B}_{1}, \ldots, \mathscr{B}_{k}\right]$ denotes the subgroup of $\mathscr{P}_{f}(\mathbf{L})$ generated by the translates of these subsets.

Corollary 3.3. Let $H^{\prime}$ and $H^{\prime \prime}$ be any two ferromagnetic translation invariant finite range interactions and assume that $D:=$ g.c.d. $\mathscr{B}\left(H^{\prime}\right)$ is factorizable. Then for all small enough $\varepsilon \geqq 0$,

$$
\varrho_{H^{\prime}+\varepsilon H^{\prime \prime}}^{+}\left(\sigma_{A}\right)=0 \quad \text { if } \quad A \notin\left[D, \mathscr{B}\left(H^{\prime \prime}\right)\right] .
$$

Proof. Since $\mathscr{B}\left(H^{\prime}\right) \subset[D]$, by Theorem 3.1 there is a constant $a>0$ such that for any positive $\varepsilon$ and any finite $A \subset \mathbf{L}$

$$
0 \leqq \varrho_{H^{\prime}+\varepsilon H^{\prime \prime}}^{+}\left(\sigma_{A}\right) \leqq \varrho_{a H_{D}+\varepsilon H^{\prime \prime}}^{+}\left(\sigma_{A}\right) .
$$

By Theorem 3.2 for all small enough $\varepsilon$ the Gibbs state of $a H_{D}+\varepsilon H^{\prime \prime}$ is unique and hence by $(2.5) \varrho_{a H_{D}+\varepsilon H^{\prime \prime}}^{+}\left(\sigma_{A}\right)=0$ if $A \notin\left[D, \mathscr{B}\left(H^{\prime \prime}\right)\right]$. Now the corollary follows from the inequality (3.7).

\subsection{Construction of Spin $\frac{1}{2}$ Models with Several Phase Transitions}

We will show now how the preceding corollary can be used to prove that there are several phase transitions in suitably constructed models.

Theorem 3.4. Let $H_{1}, \ldots, H_{n}$ be ferromagnetic translation invariant finite range interactions, let $\mathbf{B}_{i}$ be the family of the bonds of $H_{i}$, and let

$$
D_{i}=\text { g.c.d. }\left(\mathbf{B}_{1}, \ldots, \mathbf{B}_{i}\right), \quad i=1, \ldots, n .
$$

Assume furthermore that

$$
D_{1} \notin\left[\mathbf{B}_{1}, \ldots, \mathbf{B}_{n}\right] \text { and } D_{i} \notin\left[D_{i-1}, \mathbf{B}_{i}, \ldots, \mathbf{B}_{n}\right], \quad i=2, \ldots, n,
$$

and that all $D_{i}, i=1, \ldots, n$, are factorizable. Then there exist $\alpha_{1}, \ldots, \alpha_{n}>0$ such that the Hamiltonian

$$
H=\alpha_{1} H_{1}+\alpha_{2} H_{2}+\ldots \alpha_{n} H_{n}
$$

has at least $n$ phase transitions. More precisely, there exist inverse temperatures $\beta_{1}, \ldots, \beta_{n}$ such that $0<\beta_{1}<\ldots<\beta_{n}$ and

$$
\varrho_{\beta H}^{+}\left(\sigma_{D_{i}}\right)=0 \text { for } \beta<\beta_{i} \text {, and } \varrho_{\beta H}^{+}\left(\sigma_{D_{i}}\right) \neq 0 \text { for } \beta>\beta_{i} \text {. }
$$

Remarks. 1. We introduced $\alpha_{1}$ for the sake of symmetry. Rescaling, one can set $\alpha_{1}=1$, as is done in the following.

2. The theorem proves a perturbative version of a conjecture by Holsztynski [22] which states that in case $J\left(B_{i}\right)=1$ there are precisely $n$ changes of $\mathscr{A}_{\beta}$ if 
$\alpha_{1}<\alpha_{2}<\ldots<\alpha_{n}$. Numerical results on the "benchmark model" seem to contradict Holsztynski's conjecture in its original form. Also, we do not know if the only possible changes of $\mathscr{A}_{\beta}$ are those associated with splitting of the Hamiltonian, as in the theorem.

Proof. The proof has an inductive character. We will go through more initial steps than is logically necessary to facilitate the reading and to have a complete proof for the "benchmark model" before the induction starts.

By the uniqueness of the Gibbs state at high temperatures, by (2.5), and by (3.8), there exists $\beta_{1}^{\prime}$ such that

$$
\varrho_{\beta\left(H_{1}+\ldots+H_{n}\right)}^{+}\left(\sigma_{D_{1}}\right)=0 \text { for } \beta<\beta_{1}^{\prime} .
$$

By (2.8), there exists $\beta_{1}^{\prime \prime}$ such that

$$
\varrho_{\beta H_{1}}^{+}\left(\sigma_{D_{1}}\right) \neq 0 \text { for } \beta>\beta_{1}^{\prime \prime},
$$

hence, by GKS inequalities

$$
\varrho_{\beta\left(H_{1}+\ldots+H_{n}\right)}^{+}\left(\sigma_{D_{1}}\right) \neq 0 \text { for } \beta>\beta_{1}^{\prime \prime} .
$$

Therefore the model with Hamiltonian $H_{1}+\ldots+H_{n}$ exhibits at least one phase transition. To obtain a second phase transition we consider the Hamiltonians $H_{1}+\varepsilon_{2}\left(H_{2}+\ldots+H_{n}\right)$. By the previous argument there is one phase transition for any $\varepsilon_{2} \geqq 0$ with order parameter $\sigma_{D_{1}}$. We then pick a $\beta_{2}^{\prime}>\beta_{1}^{\prime \prime}$ and apply Corollary 3.3 to $H^{\prime}=\beta_{2}^{\prime} H_{1}$ and $H^{\prime \prime}=\beta_{2}^{\prime}\left(H_{2}+\ldots+H_{n}\right)$ to conclude that there exists $\bar{\varepsilon}_{2}, 0<\bar{\varepsilon}_{2}<1$, such that

$$
\varrho_{\beta_{2}^{\prime}\left(H_{1}+\bar{\varepsilon}_{2}\left(H_{2}+\ldots+H_{n}\right)\right)}^{+}\left(\sigma_{A}\right)=0 \text { if } A \notin\left[D_{1}, \mathbf{B}_{2}, \ldots, \mathbf{B}_{n}\right] .
$$

By GKS we have that

$$
\varrho_{\beta\left(H_{1}+\bar{\varepsilon}_{2}\left(H_{2}+\ldots+H_{n}\right)\right)}^{+}\left(\sigma_{A}\right)=0 \quad \text { if } \quad \beta \leqq \beta_{2}^{\prime}
$$

for all $A \notin\left[D_{1}, \mathbf{B}_{2}, \ldots, \mathbf{B}_{n}\right]$; in particular for $A=D_{2}$.

We now repeat the above construction with a slight variation. An inverse temperature $\beta_{2}^{\prime \prime}>\beta_{2}^{\prime}$ is chosen in such a way that

$$
\varrho_{\beta\left(H_{1}+\bar{\varepsilon}_{2} H_{2}\right)}^{+}\left(\sigma_{D_{2}}\right)>0 \text { for } \beta>\beta_{2}^{\prime \prime} .
$$

Such a $\beta_{2}^{\prime \prime}$ exists by (2.8). Then by GKS inequalities,

$$
\varrho_{\beta\left(H_{1}+\bar{\varepsilon}_{2}\left(H_{2}+\ldots+H_{n}\right)\right)}^{+}\left(\sigma_{D_{2}}\right) \neq 0 \text { if } \beta>\beta_{2}^{\prime \prime} ;
$$

and the model has a second phase transition associated to the order parameter $\sigma_{D_{2}}$. The requirement $\beta_{2}^{\prime}>\beta_{1}^{\prime \prime}$ implies that for the chosen $\bar{\varepsilon}_{2}$ both phase transitions occur at different temperatures.

To obtain a model with a third phase transition we resort to Hamiltonians of the form $H_{1}+\bar{\varepsilon}_{2}\left[H_{2}+\varepsilon_{3}\left(H_{3}+\ldots+H_{n}\right)\right]$ for $\varepsilon_{3} \geqq 0$. Reasoning as above we conclude that these models have two phase transitions corresponding to the order parameters $\sigma_{D_{1}}$ and $\sigma_{D_{2}}$. We pick any $\beta_{3}^{\prime}>\beta_{2}^{\prime \prime}$ and apply Corollary 3.3 to $H^{\prime}=\beta_{3}^{\prime}\left(H_{1}+\varepsilon_{2} H_{2}\right)$ and $H^{\prime \prime}=\beta_{3}^{\prime} \varepsilon_{2}\left(H_{3}+\ldots+H_{n}\right)$. We find that there exists 
$\left.\bar{\varepsilon}_{3} \in\right] 0,1[$ such that

$$
\varrho_{\beta\left(H_{1}+\bar{\varepsilon}_{2} H_{2}+\bar{\varepsilon}_{2} \bar{\varepsilon}_{3}\left(H_{3}+\ldots+H_{n}\right)\right)}^{+}\left(\sigma_{A}\right)=0 \quad \text { if } \quad \beta<\beta_{3}^{\prime}
$$

for all $A \notin\left[D_{2}, \mathbf{B}_{3}, \ldots, \mathbf{B}_{n}\right]$, in particular for $A=D_{3}$. By (2.8) we can choose $\beta_{3}^{\prime \prime}>\beta_{3}^{\prime}$ in such a way that

$$
\varrho_{\beta\left(H_{1}+\bar{\varepsilon}_{2} H_{2}+\bar{\varepsilon}_{2} \bar{\varepsilon}_{3} H_{3}\right)}^{+}\left(\sigma_{D_{3}}\right)>0 \text { for } \quad \beta>\beta_{3}^{\prime \prime},
$$

and hence, by GKS inequalities,

$$
\varrho_{\beta\left(H_{1}+\bar{\varepsilon}_{2} H_{2}+\bar{\varepsilon}_{2} \bar{\varepsilon}_{3}\left(H_{3}+\ldots+H_{n}\right)\right)}^{+}\left(\sigma_{D_{3}}\right) \neq 0 \text { for } \beta>\beta_{3}^{\prime \prime} .
$$

This proves the existence of the third transition line. It is certainly different from the previous two since $\beta_{3}^{\prime}>\beta_{2}^{\prime \prime}$.

It is now clear that by induction with respect to $k \leqq n$ we can define $\beta_{i}^{\prime}, \beta_{i}^{\prime \prime}$, and $\bar{\varepsilon}_{i}$ satisfying

$$
0<\beta_{1}^{\prime}<\beta_{1}^{\prime \prime}<\beta_{2}^{\prime}<\beta_{2}^{\prime \prime}<\ldots<\beta_{k}^{\prime}<\beta_{k}^{\prime \prime} \text { and } 0<\bar{\varepsilon}_{2}<\ldots<\bar{\varepsilon}_{k}<1,
$$

and such that for $\beta \leqq \beta_{k}^{\prime}$

$$
\varrho_{\beta\left(H_{1}+\bar{\varepsilon}_{2} H_{2}+\bar{\varepsilon}_{2} \bar{\varepsilon}_{3} H_{3}+\ldots+\bar{\varepsilon}_{2} \cdot \ldots \cdot \bar{\varepsilon}_{k-1} H_{k-1}+\bar{\varepsilon}_{2} \cdot \ldots \cdot \bar{\varepsilon}_{k}\left(H_{k}+\ldots+H_{n}\right)\right)}^{+}\left(\sigma_{A}\right)=0
$$

if $A \notin\left[D_{k-1}, \mathbf{B}_{k}, \ldots, \mathbf{B}_{n}\right]$, and for $\beta \geqq \beta_{k}^{\prime \prime}$

$$
\varrho_{\beta\left(H_{1}+\bar{\varepsilon}_{2} H_{2}+\bar{\varepsilon}_{2} \bar{\varepsilon}_{3} H_{3}+\ldots+\bar{\varepsilon}_{2} \cdot \ldots \cdot \bar{\varepsilon}_{k-1} H_{k-1}+\bar{\varepsilon}_{2} \cdot \ldots \cdot \bar{\varepsilon}_{k} H_{k}\right)}^{+}\left(\sigma_{D_{k}}\right) \neq 0 .
$$

Now, let

$$
\alpha_{1}=1, \quad \alpha_{2}=\bar{\varepsilon}_{2}, \quad \alpha_{3}=\bar{\varepsilon}_{2} \bar{\varepsilon}_{3}, \ldots, \alpha_{n}=\bar{\varepsilon}_{2} \bar{\varepsilon}_{3} \cdot \ldots \cdot \bar{\varepsilon}_{n}
$$

and

$$
H=\alpha_{1} H_{1}+\alpha_{2} H_{2}+\ldots+\alpha_{n} H_{n}
$$

since $0<\varepsilon_{i}<1$, the Hamiltonian in (3.12) is "more ferromagnetic" than $H$ and therefore (by GKS) for $\beta \leqq \beta_{k}^{\prime}$

$$
\varrho_{\beta H}^{+}\left(\sigma_{A}\right)=0 \text { if } A \notin\left[D_{k-1}, \mathbf{B}_{k}, \ldots, \mathbf{B}_{n}\right],
$$

and in particular, by (3.8),

$$
\varrho_{\beta H}^{+}\left(\sigma_{D_{k}}\right)=0 \text { for } \beta \leqq \beta_{k}^{\prime} .
$$

Similarly, since the Hamiltonian in (3.13) is "less ferromagnetic" than $H$, again by GKS

$$
\varrho_{\beta H}^{+}\left(\sigma_{D_{k}}\right) \neq 0 \text { for } \quad \beta \geqq \beta_{k}^{\prime \prime} \text {. }
$$

Let

$$
\beta_{k}=\inf \left\{\beta: \varrho_{\beta H}^{+}\left(\sigma_{D_{k}}\right) \neq 0\right\}
$$

by GKS (3.9) holds, and since $\beta_{i}^{\prime} \leqq \beta_{i} \leqq \beta_{i}^{\prime \prime}$ and $\beta_{i+1}^{\prime}>\beta_{i}^{\prime \prime}$ one sees that $\beta_{i+1}$ is strictly larger than $\beta_{i}$. This finishes the proof. 
The following corollary yields a concrete class of examples, of which the "benchmark model" is a particular case.

Corollary 3.5. If g.c.d. $(D, A)=1,1 \notin[D, A]$ and $D$ is factorizable then for any $n$ there exist $\alpha_{2}, \ldots, \alpha_{n}>0$ such that

$$
H=H_{D^{n}}+H_{D^{n-1} \cdot A}+\alpha_{2} H_{D^{n-2} \cdot A}+\ldots+\alpha_{n-1} H_{D \cdot A}+\alpha_{n} H_{A}
$$

exhibits at least $n$ phase transitions. More precisely, there exist $0<\beta_{1}<\beta_{2}<\ldots<\beta_{n}$ such that

$$
\varrho_{\beta H}^{+}\left(\sigma_{D^{n-i}}\right)=0 \text { for } \beta<\beta_{i}, \quad \text { and } \varrho_{\beta H}^{+}\left(\sigma_{D^{n-i}}\right) \neq 0 \text { for } \beta>\beta_{i},
$$

where $D^{0}=1=\{0\}$.

Proof. All one has to check is that $D_{i}=D^{n-i}$ and that the condition (3.8) is satisfied.

We apply the theorem to $H_{1}=H_{D^{n}}+H_{D^{n-1} \cdot A}, H_{2}=H_{D^{n-2} \cdot A}, \ldots, H_{n-1}=H_{D \cdot A}$ and $H_{n}=H_{A}$. We have: $D_{1}=$ g.c.d. $\left(\mathbf{B}_{1}\right)=$ g.c.d. $\left(D^{n}, D^{n-1} \cdot A\right)=D^{n-1}$ and $D^{n-1} \notin\left[D^{n}, D^{n-1} \cdot A\right]$, since otherwise $1 \in[D, A]$. Similarly, $D_{2}=$ g.c.d. $\left(\mathbf{B}_{1}, \mathbf{B}_{2}\right)$ $=$ g.c.d. $\left(D^{n-1}, D^{n-2} \cdot A\right)=D^{n-2}$ and again $D^{n-2} \notin\left[D^{n-1}, D^{n-2} \cdot A\right]$. It should be now obvious that the claim follows from the theorem.

Remark. Both by inspection of the proof and by a continuity argument one can see that if the theorem, or the corollary, holds for some values $\alpha_{i}$ of the parameters it holds for all values of the parameters in some neighborhood of $\alpha_{i}$.

\subsection{Proof of the Inequality (Theorem 3.1)}

By the hypothesis (3.1) of Theorem 3.1 each bond of $\mathscr{B}_{1}$ is a symmetric difference of bonds of $\mathscr{B}_{2}$. This implies that there exists a map from $\mathscr{B}_{1}$ to finite subsets of $\mathscr{B}_{2}$, $B \mapsto \mathscr{C}_{B}$, which commutes with translations and such that

$$
\prod_{C \in \mathscr{C}_{B}} \sigma_{C}=\sigma_{B} .
$$

Moreover, we can choose the families $\mathscr{C}_{B}$ in such a way that $\mathscr{C}_{B}$ contains no nontrivial cycle, i.e.

$$
\mathscr{K}\left(\mathscr{C}_{B}\right)=\{\emptyset\} .
$$

Furthermore, for each $C \in \mathscr{B}_{1}$ we define an interaction $J_{B}$ with bonds $\mathscr{C}_{B}$ in such a way that $B \mapsto J_{B}$ commutes with translations and

$$
\sum_{B: C \in \mathscr{C}_{B}} J_{B}(C) \leqq J_{2}(C)
$$

for any $C \in \mathscr{B}_{2}$. For any $B \in \mathscr{B}_{1}$ we now set

$$
\Phi_{B}=\sum_{C \in \mathscr{C}_{B}} J_{B}(C) \sigma_{C} ;
$$

$\left(\Phi_{B}\right)_{B \in \mathscr{B}_{1}}$ is a translation invariant finite range interaction in an obvious sense. Note, however, that $\Phi_{B}(X)$ depends on the restriction of the configuration $X$ to

$$
s(B)=\bigcup_{C \in \mathscr{C}_{B}} C
$$

which may be larger than $B$. 
Because of (3.15) and the second GKS inequality, it is enough to prove the theorem with $\mathrm{H}_{2}$ replaced by

$$
H_{2}^{\prime}=-\sum_{B} \Phi_{B}
$$

This is what we will do.

We consider a finite subset $\Lambda$ of the lattice. We let $L_{1}$ and $L_{2}$ denote the Hamiltonians in $\Lambda$ defined by $\beta H_{1}+K$ and $f(\beta) H_{2}^{\prime}+K$, respectively, and by the " 1 "-boundary conditions in the complement of $\Lambda$. Let $Z_{1}$ and $Z_{2}$ be the corresponding partition functions. Our goal is to define $f(\beta)$ such that

$$
\left(Z_{2}\right)^{-1} \sum_{X \in \mathbf{X}_{A}} \sigma_{A}(X) e^{-L_{2}(X)}-\left(Z_{1}\right)^{-1} \sum_{X \in \mathbf{X}_{A}} \sigma_{A}(X) e^{-L_{1}(X)} \geqq 0
$$

for every $A$ and every $A \subset A$. This is equivalent, [17], to

$$
\begin{aligned}
0 \leqq & \left(Z_{1} Z_{2}\right)^{-1} \sum_{X, Y \in \mathbf{x}_{A}}\left[\sigma_{A}(X)-\sigma_{A}(Y)\right] e^{-L_{2}(X)-L_{1}(Y)} \\
& =\left(Z_{1} Z_{2}\right)^{-1} \sum_{X, Y \in \mathbf{X}_{A}}\left[1-\sigma_{A}(Y)\right] \sigma_{A}(X) e^{-L_{2}(X)-L_{1}(X \cdot Y)},
\end{aligned}
$$

where the last line was obtained by making the change of variables $Y \mapsto X \cdot Y$, $X \mapsto X$. Since $1-\sigma_{A}(Y) \geqq 0$, it is enough to show that

$$
\sum_{X} \sigma_{A}(X) e^{-L_{2}(X)-L_{1}(X \cdot Y)} \geqq 0
$$

for each $Y \in \mathbf{X}_{\Lambda}$. That is, we must show that

$$
\begin{aligned}
& e^{-L_{2}(X)-L_{1}(X \cdot Y)} \\
& =\exp \left[f(\beta) \sum_{B: s(B) \cap A \neq \emptyset} \Phi_{B}(X \vee 1)+\beta \sum_{B: B \cap A \neq \emptyset} J_{1}(B) \sigma_{B}(X \cdot Y)\right. \\
& \left.\quad+\sum_{B: B \cap A \neq \emptyset} J_{3}(B)\left[1+\sigma_{B}(Y)\right] \sigma_{B}(X)\right]
\end{aligned}
$$

is a positive definite (p.d.) function of $X$ for each $Y \in \mathbf{X}_{A}$; here $X \vee 1$ denotes the configuration equal to $X$ on $A$ and to 1 on the complement of $\Lambda$. Up to this point we followed Ginibre's [17] proof of one of the GKS inequalities.

Now, (3.16) can be written as a product of factors of the form

$$
\begin{gathered}
\exp \left[\sum_{B: B \cap A \neq \emptyset} J_{3}(B)\left[1+\sigma_{B}(Y)\right] \sigma_{B}(X)\right], \\
\exp \left[f(\beta) \Phi_{B}(X \vee 1)\right] \quad \text { with } \quad s(B) \cap \Lambda \neq \emptyset, \quad B \cap \Lambda=\emptyset,
\end{gathered}
$$

and

$$
\exp \left[f(\beta) \Phi_{B}(X \vee 1)+\beta J_{1}(B) \sigma_{B \cap A}(X \cdot Y)\right] \quad \text { with } \quad B \cap \Lambda \neq \emptyset .
$$

Expressions (3.17) and (3.18) are p.d. functions of $X$ because they are exponentials of p.d. functions (see [17, first GKS]). Thus it is enough to prove that one can choose $f$ in such a way that (3.19) is p.d.

We note first that it is enough to consider the case of $s(B)$ contained in $A$. For otherwise the function (3.19) of $X$ can be considered as a restriction to 
$\mathbf{X}_{\Lambda} \times\left\{1_{\Lambda^{\prime} \backslash \Lambda}\right\}$, where $\Lambda^{\prime} \supset \Lambda$ is big enough, of the function

$$
X \mapsto \exp \left[f(\beta) \sum_{C \in \mathscr{C}_{B}} J_{B}(C) \sigma_{C}(X)+\beta J_{1}(B) \sigma_{B}(X \cdot Y)\right] .
$$

And since a restriction of a p.d. function to a subgroup is p.d., our claim follows. Thus we reduced the problem to showing that

$$
R(A) \equiv \sum_{X \in \mathbf{X}_{A}} \sigma_{A}(X) \exp \left[f(\beta) \sum_{C \in \mathscr{C}_{B}} J_{B}(C) \sigma_{C}(X)+\beta J_{1}(B) \sigma_{B}(X \cdot Y)\right] \geqq 0
$$

for each finite subset $A$ of the lattice, $A \subset A$, and each $B$ such that $C \subset A$ for all $C \in \mathscr{C}_{B}$. This we do by resorting to the $\operatorname{HTE}(2.12)$.

According to (2.12), $R(A)=0$ if $A \notin \overline{\{B\} \cup \mathscr{C}_{B}}=\overline{\mathscr{C}_{B}}$. Hence it is enough to consider the case of $A \in \overline{\mathscr{C}}_{B}$, that is, of such $A \subset A$ for which there is an $\alpha_{A} \subset \mathscr{C}_{B}$ such that $\sigma_{A}=\prod_{C \in \alpha_{A}} \sigma_{C}$ [since $\mathscr{K}\left(\mathscr{C}_{B}\right)=0$ such $\alpha_{A}$ is unique]. The HTE of $R(A)$ involves a sum over all cycles $\alpha \in \mathscr{K}\left(\mathscr{C}_{B} \cup\{B\}\right)$. By (3.14), there are exactly two such cycles: the trivial one, $\alpha=\emptyset$, and the one formed by all the bonds, $\alpha=\{B\} \cup \mathscr{C}_{B}$. Therefore, the HTE of $R(A)$ for $A=\overline{\alpha_{A}}$ is

$$
\begin{aligned}
& 2^{|\Lambda|} \cosh \left[\beta J_{1}(B) \sigma_{B}(Y)\right]\left[\prod_{C \in \mathscr{C}_{B}} \cosh \left[f(\beta) J_{B}(C)\right]\right] \\
& \quad \times\left[\prod_{C \in \alpha_{A}} \tanh \left[f(\beta) J_{B}(C)\right]+\tanh \left[\beta J_{1}(B) \sigma_{B}(Y)\right] \prod_{C \in \mathscr{C}_{B} \backslash \alpha_{A}} \tanh \left[f(\beta) J_{B}(C)\right]\right] .
\end{aligned}
$$

Since $|\tanh x|<1$, we see that $R(A)$ is made non-negative choosing $f(\beta)$ in such a way that

$$
\prod_{C \in \mathscr{C}_{B}} \tanh \left[f(\beta) J_{B}(C)\right] \geqq\left|\tanh \beta J_{1}(B) \sigma_{B}(Y)\right|=\left|\tanh \beta J_{1}(B)\right|
$$

for each $B \in \mathscr{B}_{1}$. Since $J_{1}$ is translation invariant and of a finite range one has here a finite number of inequalities which certainly admit a solution $f$ satisfying the condition (3.2). This ends our proof of Theorem 3.1.

We note that the condition that $H_{1}$ be ferromagnetic is not essential. In the general case $\varrho^{+}$of the left-hand side of (3.3) can be replaced by any Gibbs state of $\beta H_{1}+K$. We also note that our proof shows that for the Hamiltonian $\beta H_{1}+f(\beta) H_{2}+K$ both the first and the second GKS inequality hold, and that one obtains the following stability property of the inequalities: if $\mathrm{H}_{2}$ is ferromagnetic then for any small enough $H_{1}$, ferromagnetic or not, for which $\mathscr{B}\left(H_{1}\right) \subset \overline{\mathscr{B}\left(H_{2}\right)}$, the GKS inequalities hold for $H_{1}+H_{2}$.

\section{The Uniqueness Result}

This section is devoted to the proof of Theorem 3.2 using a criterion due to Dobrushin and Shlosman [8] (DS-criterion) which we review and slightly adapt in the sequel. In this section we absorb $\beta$ in the definition of the interaction.

\subsection{The Basic Criterion}

Let us introduce the notation needed to state DS-criterion. For a separable metric space $\mathbf{X}$ with metric $\delta$ let $R_{\delta}$ be the Kantorovich-Wasserstein metric on the space of 
measures on $\mathbf{X}$ :

$$
R_{\delta}(\mu, \varrho)=\sup _{|f|_{L} \leqq 1}|\mu(f)-\varrho(f)|,
$$

where the Lipschitz seminorm $|\cdot|_{L}$ on the space of real-valued measurable functions is:

$$
|g|_{L}=\sup _{X \neq Y} \frac{|f(X)-f(Y)|}{\delta(X, Y)}
$$

(Definition (4.1) can be stated equivalently [10, lecture 20] in terms of joint distributions.) In particular, if $\mathbf{X}$ is finite and $\chi$ is the discrete metric $\chi(X, Y)=1-\delta_{X, Y}$; the metric (4.1) coincides with the variational metric

$$
R_{\chi}(\mu, \varrho)=\operatorname{Var}(\mu, \varrho) \equiv \sup _{A \text { measurable }}|\mu(A)-\varrho(A)| .
$$

DS-criterion consists in a bound on the Kantorovich-Wasserstein distance for Gibbs measures on a finite configuration space $\mathbf{X}_{\Lambda}$ with metric

$$
\delta_{\Lambda}(Y, Z)=\sum_{t \in \Lambda} \chi\left(Y_{t}, Z_{t}\right)
$$

We remark that then

$$
R_{\delta_{\Lambda}}(\mu, \varrho) \leqq|\Lambda| \operatorname{Var}(\mu, \varrho)
$$

for any measures $\mu, \varrho$ on $\mathbf{X}_{\Lambda}$.

We consider the space $\widetilde{A}_{r}$ of interactions of a fixed range $r<\infty$, with the norm $|\phi|=\sup _{B \in \mathscr{B}}\left|\phi_{B}\right|_{\infty}$, and for a finite $\Lambda \subset \mathbf{Z}^{v}$ we denote $\partial \Lambda=\left\{x \in \Lambda^{c}: \operatorname{dist}(x, \Lambda)<r\right\}$. We then have:

Theorem 4.1 (Dobrushin-Shlosman). Assume that there exists a finite $\Lambda \subset \mathbf{Z}^{v}$ such that for all pairs of configurations $X, Z$ differing at most at one point

$$
R_{\delta_{\Lambda}}\left(\varrho_{\Lambda}(\cdot \mid Y), \varrho_{\Lambda}(\cdot \mid Z)\right) \leqq \frac{(1-\varepsilon)|\Lambda|}{|\partial \Lambda|}
$$

for some $\varepsilon>0$. Then for all the interactions in an open nonempty neighborhood of $\phi$ in $\tilde{A}_{r}$, the Gibbs state is unique.

We will use a sufficient condition for this theorem almost identical to Theorem 3.3 of [8]. For finite sets $\Lambda \subset M \subset \mathbf{Z}^{v}$ and a (partial) configuration $Y$ we denote by $\varrho_{M, A}(\cdot \mid Y)$ the projection of $\varrho_{M}(\cdot \mid Y)$ on $\mathbf{X}_{\Lambda}$ :

$$
\varrho_{M, \Lambda}(f \mid Y)=Z(M \mid Y)^{-1} \int_{\mathbf{x}_{\Lambda}} d X \int_{\mathbf{x}_{M \backslash \Lambda}} d Z f(X) e^{-H_{M}(X, Z \mid Y)} .
$$

In addition we consider two integers $n, d$ which will be chosen eventually large enough and such that $n \gg d$. The integer $n$ is the size of the side of a (large) cube $D_{n}=\left\{t \in \mathbf{Z}^{v}: 0 \leqq t^{i} \leqq n, i=1, \ldots, v\right\}$, while $d$ is the size of much smaller cubes of "excluded" volume placed inside $D_{n}$ adjacent to its boundary. These cubes are defined as follows. We take $d$ in the range $2 r<d<n / 3$ and for each $t_{0} \in \partial D_{n}$ we 

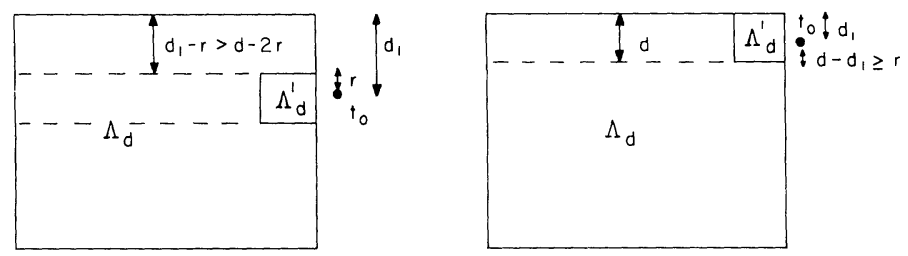

Fig. 1. Possible choices of the cubes $\Lambda_{d}^{\prime}\left(t_{0}\right)$ for a site $t_{0}$ at a distance $d_{1}$ from an edge of a parallelepiped $D_{n}$. The figure on the left corresponds to the case $d_{1}>d-r$, while the one on the right to $d_{1} \leqq d-r$. The dashed lines represent the boundary of the parallelepipeds $T_{i}$ of the decomposition (4.7). Each $\Lambda_{d}^{\prime}\left(t_{0}\right)$ is chosen so that the point $t_{0}$ is at least a distance $r$ away from $\Lambda_{d}\left(t_{0}\right)$ and all sides of the parallelepipeds $T_{i}$ are at least of size $d-2 r$

choose a cube $\Lambda_{d}^{\prime}\left(t_{0}\right) \subset D_{n}$ of side of length $d$ such that, if $\Lambda_{d}\left(t_{0}\right) \equiv D_{n} \backslash \Delta_{d}^{\prime}\left(t_{0}\right)$ we have:

C1) No spin in $\Lambda_{d}\left(t_{0}\right)$ interacts with the spin at $t_{0}$, i.e. $\left|t-t_{0}\right|>r$ for all $t \in \Lambda_{d}\left(t_{0}\right)$.

C2) The set $\Lambda_{d}\left(t_{0}\right)$ can be decomposed into a disjoint union of a finite number $k$ of large enough parallelepipeds $T_{i}$; explicitly:

$$
\Lambda_{d}\left(t_{0}\right)=\bigcup_{i=1}^{k} T_{i}, \quad 1 \leqq i \leqq k
$$

where $k=k\left(\Lambda_{d}\right)$ does not exceed a certain integer $p(v)$ that depends only on the dimension, and each $T_{i}$ has sides of size at least $d-2 r$.

Such a choice is always possible (see Fig. 1). In the sequel the $t_{0}$ dependence of the sets $\Lambda_{d}$ and $\Lambda_{d}^{\prime}$ will not be written explicitly in order to simplify the notation, which we try to keep close to that of [8].

Our proof of Theorem 3.2 is based on a slight adaptation of a theorem by Dobrushin and Shlosman:

Lemma 4.2. If for $n, d$ large enough with $2 r<d<n / 3$ and each $t_{0} \in \partial D_{n}$,

$$
\operatorname{Var}\left(\varrho_{D_{n}, \Lambda_{d}}(\cdot \mid Y), \varrho_{D_{n}, \Lambda_{d}}(\cdot \mid W)\right) \leqq c e^{-\gamma d}
$$

for some $c, \gamma>0$, for each pair of configurations $Y, W$ coinciding off $t_{0}$; then (4.6) and therefore also the conclusions of Theorem 4.1 hold.

Remark. This result is basically proven in [8] (Theorem 3.3). For the sake of completeness we present an alternative proof based on the expression (4.1) for the Kantorovich-Wasserstein metric.

Proof. Choose a $t_{0} \in D_{n}$ and consider a pair of configurations $Y, W$ coinciding off $t_{0}$. Take an arbitrary measurable function $f$ on $D_{n}$ with $|f|_{L} \leqq 1$ for the distance $\delta_{D_{n}}$ [defined in (4.4)], i.e., such that

$$
|f(X)-f(Y)| \leqq\left|\left\{i \in M: X_{i} \neq Y_{i}\right\}\right| .
$$

The idea is to write the average of $f$ as the sum of two contributions: one due to the averaging over configurations inside $\Lambda_{d}$ and the part corresponding to flippings of spins in $\Lambda_{d}^{\prime}$. The former is insensitive to the boundary conditions by hypothesis (4.8); and the latter contribution can be bounded by the sum of the 
oscillations of $f$ in $\Lambda_{d}^{\prime}$ - which is a small correction if the volume of $\Lambda_{d}^{\prime}$ is negligible compared to that of $D_{n}$.

Let us then define a new function $f^{*}$-which can be identified with a function on $\mathbf{X}_{\Lambda_{d}}$ - obtained by fixing the spins in $\Lambda_{d}^{\prime}$ at some value $g$. That is, if $g_{A_{d}^{\prime}}$ denotes the configuration that at each site takes value $g$ :

$$
f^{*}(X)=f\left(X_{\Lambda_{d}}, g_{\Lambda_{d}^{\prime}}\right) .
$$

Obviously

$$
\left|f^{*}\right|_{L} \leqq|f|_{L} \leqq 1
$$

We have:

$$
\begin{aligned}
& \left|\varrho_{D_{n}}(f \mid Y)-\varrho_{D_{n}}(f \mid W)\right| \\
& \quad \leqq \\
& \quad \varrho_{D_{n}}(f \mid Y)-\varrho_{D_{n}}\left(f^{*} \mid Y\right)|+| \varrho_{D_{n}}\left(f^{*} \mid Y\right)-\varrho_{D_{n}}\left(f^{*} \mid W\right) \mid \\
& \quad+\left|\varrho_{D_{n}}\left(f^{*} \mid W\right)-\varrho_{D_{n}}(f \mid W)\right|
\end{aligned}
$$

which implies

$$
\begin{aligned}
& \left|\varrho_{D_{n}}(f \mid Y)-\varrho_{D_{n}}(f \mid W)\right| \leqq 2\left|f-f^{*}\right|_{\infty} \\
& \quad+\sup \left\{\left|\varrho_{D_{n}}(h \mid Y)-\varrho_{D_{n}}(h \mid W)\right|: h \text { on } \mathbf{X}_{\Lambda_{d}} \text { with }|h|_{L} \leqq 1\right\} .
\end{aligned}
$$

If $X$ is the configuration for which $\left|f-f^{*}\right|_{\infty}$ is achieved, we obtain from (4.9) and (4.10):

$$
\left|f-f^{*}\right|_{\infty}=\left|f(X)-f\left(X_{\Lambda_{d}}, g_{\Lambda_{d}^{\prime}}\right)\right| \leqq\left|\Lambda_{d}^{\prime}\right| .
$$

On the other hand, the second term in (4.12) is precisely

$$
\begin{aligned}
R_{\delta_{A}}\left(\varrho_{D_{n}, \Lambda_{d}}(\cdot \mid Y), \varrho_{D_{n}, \Lambda_{d}}(\cdot \mid Z)\right) & \leqq\left|\Lambda_{d}\right| \operatorname{Var}\left(\varrho_{D_{n}, \Lambda_{d}}(\cdot \mid Y), \varrho_{D_{n}, \Lambda_{d}}(\cdot \mid Z)\right) \\
& \leqq\left|\Lambda_{d}\right| c e^{-\gamma d} .
\end{aligned}
$$

The first inequality is due to (4.5) and the second one to the hypothesis (4.8). If the bounds (4.13) and (4.14) are substituted into (4.12) we obtain:

$$
\begin{aligned}
R_{\delta_{D_{n}}}\left(\varrho_{D_{n}, \Lambda_{d}}(\cdot \mid Y), \varrho_{D_{n}, \Lambda_{d}}(\cdot \mid W)\right) & \leqq 2\left|\Lambda_{d}^{\prime}\right|+c\left|\Lambda_{d}\right| e^{-\gamma d} \\
& \leqq 2 d^{v}+c^{\prime} n^{v} e^{-\gamma d}
\end{aligned}
$$

uniformly in $t_{0}$. It follows that if one chooses $d^{v}=\varepsilon n$ with $\varepsilon<1 / 2$ the condition (4.6) is satisfied for $\Lambda=D_{n}$ with $n$ large enough.

Furthermore, condition (4.8) of Lemma 4.2 can be obtained from a bound on the reduced partition function. Indeed, as the Hamiltonian on $\Lambda_{d}$ does not depend on the value of the spin at $t_{0}$ (condition $\mathrm{C} 1$ above), it is straightforward to obtain

$$
\begin{aligned}
& \operatorname{Var}\left(\varrho_{D_{n}, \Lambda_{d}}(\cdot \mid Y), \varrho_{D_{n}, \Lambda_{d}}(\cdot \mid W)\right) \\
& \quad \leqq Z\left(\Lambda_{d} \mid Y\right) \sup _{X \in \mathbf{X}_{\Lambda_{d}}}\left|\frac{Z\left(\Lambda_{d}^{\prime} \mid Y, X\right)}{Z\left(D_{n} \mid Y\right)}-\frac{Z\left(\Lambda_{d}^{\prime} \mid W, X\right)}{Z\left(D_{n} \mid W\right)}\right| \\
& \quad=Z^{0}\left(\Lambda_{d} \mid Y\right) \sup _{X \in \mathbf{X}_{\Lambda_{d}}}\left|\frac{Z^{0}\left(\Lambda_{d}^{\prime} \mid Y, X\right)}{Z^{0}\left(D_{n} \mid Y\right)}-\frac{Z^{0}\left(\Lambda_{d}^{\prime} \mid W, X\right)}{Z^{0}\left(D_{n} \mid W\right)}\right| .
\end{aligned}
$$


Therefore, we see that to prove (4.8) it is enough to show that all the reduced partition functions in (4.16) tend to one exponentially fast with $d$ uniformly in the boundary conditions. Moreover, using the decomposition (4.7) we have the bound

$$
\inf _{\left(X_{i}\right)} \prod_{i} Z^{0}\left(T_{i} \mid X_{i}\right) \leqq Z^{0}\left(\Lambda_{d} \mid Y\right) \leqq \sup _{\left(X_{i}\right)} \prod_{i} Z^{0}\left(T_{i} \mid X_{i}\right)
$$

which shows that it is enough to prove the exponential behavior of $Z^{0}$ only on parallelepipeds. We thus have:

Proposition 4.3. If an interaction $\phi$ is such that there exist positive $\alpha$ and $\gamma$ with the property that for each parallelepiped $\Lambda=\left[0, N_{1}\right] \times \ldots \times\left[0, N_{v}\right]$ and each boundary condition $Y$

$$
\left|Z^{0}(\Lambda \mid Y)-1\right| \leqq \alpha e^{-\gamma \inf _{i} N_{i}},
$$

then (4.8) holds and hence all interactions in some open neighborhood of $\phi$ in $\tilde{A}_{r}$ have unique Gibbs states.

\subsection{Partition Functions for Factorizable Trivial Systems}

To conclude the proof of Theorem 3.2 we use the HTE to show that the bound (4.17) on $Z^{0}(\Lambda \mid Y)$ holds for factorizable trivial systems. We notice that for general spin- $\frac{1}{2}$ systems, the set of bonds defined by a given boundary condition $Y \in \mathbf{X}_{M}$, $M \subset A^{c}$, depends only on the set $M$; and the corresponding set of cycles increases with $M$. If we denote by $\mathscr{K}_{A}$ the set of cycles for the boundary condition 1 (or any boundary condition $Y \in \mathbf{X}_{A^{c}}$ ), we have from the $\operatorname{HTE}(2.10)$ that for a trivial system with Hamiltonian $H=-J \sum_{x} \sigma_{C+x}$;

$$
\left|Z^{0}(\Lambda \mid Y)-1\right| \leqq \sum_{\substack{\alpha \in \mathscr{K}_{A} \\ \alpha \neq 0}} t^{|\alpha|}
$$

with $t=\tanh |J|$.

The result (4.17) will be obtained from (4.18) plus the fact that the cycles for factorizable trivial systems are superpositions of periodic arrays of bonds parallel to the coordinate axis. Therefore each cycle involves a large number of bonds, which causes the right-hand side of (4.18) to decrease exponentially with the linear dimensions of $A$.

To state this crucial property of the cycles it is notationally convenient to identify families of bonds with subsets of $\mathbf{Z}^{v}$ :

$$
\alpha \leftrightarrow\left\{x \in \mathbf{Z}^{v}: C+x \in \alpha\right\} .
$$

We adopt such identification for the rest of this section; in particular the cycles $\alpha \in \mathscr{K}_{\Lambda}$ are identified with subsets of $\Lambda^{*}=\left\{x \in \mathbf{Z}^{v}:(C+x) \cup \Lambda \neq \emptyset\right\}$.

To follow the argument it may be useful to keep in mind a simple example (Fig. 2). Consider the system in $\mathbf{Z}^{2}$ with the unit square as fundamental bond:

$$
C=\{(0,0),(0,1),(1,1),(1,0)\} \text {. }
$$




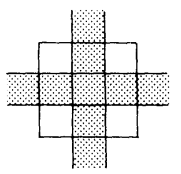

(a)

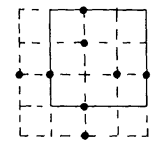

$\propto$

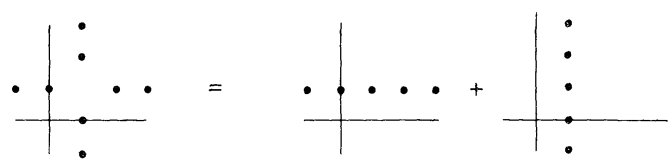

(b)
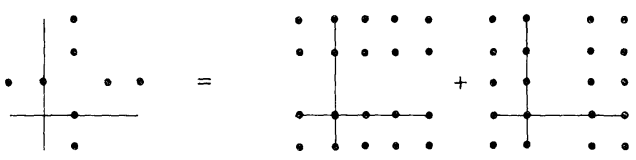

(c)

Fig. 2a-c. Part a is a cycle with fundamental bond (4.20) on a $3 \times 3$ square $A$; and, on the right, the corresponding subset of the lattice obtained through the identification (4.19). Parts $\mathbf{b}$ and $\mathbf{c}$ show two different decompositions of $\alpha$ in the form (4.21). The decomposition in part $\mathbf{b}$ is economical while that in part $\mathbf{c}$ is not. The symbol "+" stands for the symmetric difference

Its cycles - for " 1 " boundary conditions - are formed by the superposition of arrays of bonds parallel to each coordinate axis, with period 1. In the left part of Fig. 2 a we show an example of a cycle when $\Lambda$ is a three-by-three square. The corresponding set $\alpha$, obtained via (4.19), is formed by the heavy dots in the diagram of the right; where we have used dashed lines to depict the set $\Lambda^{*}$.

In the appendix we prove the following characterization of the cycles of factorizable trivial systems.

Proposition 4.4. For a factorizable trivial system there exists an integer $q$ such that every element $\alpha$ of $\mathscr{K}_{A}$ has a decomposition

$$
\alpha=\sum_{i=1}^{v} \alpha(i)
$$

where " $\sum$ " stands for the symmetric difference and each $\alpha(i)$ is a subset of $\Lambda^{*}$ periodic in the $i^{\text {th }}$ direction with period $q$. This decomposition is far from unique.

Let us fix $q$ in the sequel and denote for each set $\Lambda$ and for $1 \leqq i \leqq v$;

$L_{i, q}(\Lambda)=\left\{\alpha \subset A:\left.\alpha\right|_{\Lambda}\right.$ is periodic in the $i^{\text {th }}$ direction with period $\left.q\right\}$,

$$
L_{\leqq v}(\Lambda)=\left\{\alpha=\sum_{j=1}^{v} \alpha(j): \alpha(j) \in L_{j, q}(\Lambda)\right\} .
$$


With such notation, Proposition 4.4 can be succinctly stated as

$$
\mathscr{K}_{\Lambda} \subset L_{\leqq v}\left(\Lambda^{*}\right) .
$$

From (4.18) and (4.22) we see that to obtain the bound (4.17) for factorizable trivial systems, it is enough to prove the following proposition. (Note that if $\Lambda$ is a parallelepiped, the set $\Lambda^{*}$ is a larger parallelepiped.)

Proposition 4.5. Assume $A=\left[0, N_{1}\right] \times \ldots \times\left[0, N_{v}\right]$. Then, for each $t<1$ there exist $c_{1}, c_{2}>0$ depending on $t, q, v$ but independent of $N_{1}, \ldots, N_{v}$ such that

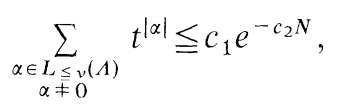

where $N=\min _{1 \leqq j \leqq \nu} N_{j}$.

Proof. We will show, by induction in $i$, that

$$
\sum_{\substack{\alpha \in L \leqq i(\Lambda) \\ \alpha \neq 0}} t^{|\alpha|} \leqq c_{1} e^{-c_{2} N},
$$

for every $1 \leqq i \leqq v$.

For $i=1, \alpha(1)$ is formed by $\left(N_{2}+1\right) \ldots\left(N_{v}+1\right)$ periodic one dimensional sets parallel to the first axis; hence

$$
\sum_{\alpha \in L_{1}(\Lambda)} t^{|\alpha|}=\left[\sum_{\alpha \in L_{1}\left(\left[0, N_{1}\right]\right)} t^{|\alpha|}\right]^{\left(N_{2}+1\right) \ldots\left(N_{v}+1\right)} .
$$

The fact that each set of $L_{1}\left(\left[0, N_{1}\right]\right)$ has period $q$ implies that there are at most $2^{q}$ different such sets, and all except the empty one have at least $N_{1} / q$ points; hence:

$$
\sum_{\alpha \in L_{1}\left(\left[0, N_{1}\right]\right)} t^{|\alpha|} \leqq 1+2^{q} t^{N_{1} / q} .
$$

The bound (4.23) for $i=1$ follows from (4.25) and (4.26).

For the inductive step we use the following lemma.

Lemma 4.6. If $2 \leqq k \leqq v$,

$$
\sum_{\alpha \in L \leqq k(\Lambda)} t^{|\alpha|} \leqq\left[\sum_{\chi \in L_{k}(\Lambda)} t^{|x| / 3}\right]\left[\sum_{\theta \in L \leqq k-1(\Lambda)} t^{|\theta| / 3}\right] .
$$

Proof. Every $b \in L_{\leqq k}(\Lambda)$ is of the form

$$
\alpha=\chi+\theta
$$

with $\chi \in L_{k}(\Lambda)$ (set of "columns") and $\theta \in L_{\leq k-1}(\Lambda)$ (set of "horizontal sections," which in Fig. 2 are in fact "rows"). The decomposition (4.28) is not unique, and to prove (4.27) we need to restrict this degeneracy and to select those decompositions for which $|\chi|+|\theta|$ does not differ too much from $|\alpha|$. That is, we need to exclude decompositions as that or part c of Fig. 2 where there are many "annihilations." For such purposes we adopt the following definition.

Definition 4.7. The decomposition (4.28) is economical if every other decomposition $\left(\chi^{\prime}, \theta^{\prime}\right)$ of the same $\alpha$ satisfies

$$
|\chi| \leqq\left|\chi^{\prime}\right| .
$$


For each element of $L_{\leqq k}(\Lambda)$ there may be several economical decompositions, hence:

$$
\sum_{\alpha \in L \leqq k(\Lambda)} t^{|\alpha|} \leqq \sum_{(\chi, \theta) \in \pi(\Lambda)} t^{|\chi+\theta|},
$$

where $\pi(\Lambda)$ denotes the set formed by all the economical decompositions of the elements of $L_{\leqq k}(\Lambda)$. From (4.30) we see that to prove (4.27) it suffices to show that for all economical decompositions

$$
|\chi+\theta| \geqq \frac{|\chi|}{3}-\frac{|\theta|}{3} .
$$

But the fact that

$$
|\chi+\theta|-\left(\frac{|\chi|}{3}-\frac{|\theta|}{3}\right)=\frac{2}{3}(|\chi|-|\chi \cap \theta|)+\frac{2}{3}(|\theta|-2|\chi \cap \theta|)
$$

shows that (4.31) - and hence (4.27) - is a consequence of the following property of economical decompositions:

Claim. For an economical decomposition $(\chi, \theta)$ :

$$
|\theta| \geqq 2|\chi \cap \theta| \text {. }
$$

Indeed, consider the "section" of $\theta$ at "height" $j$ :

$$
\theta_{j}=\theta \cap\left\{a \in \mathbf{Z}^{v}: a_{k}=j\right\} \text {. }
$$

If (4.32) were false there would exist some $j_{0}$ such that

$$
\left|\theta_{j_{0}}\right|<2\left|\chi \cap \theta_{j_{0}}\right|
$$

In such case we could define another decomposition $\left(\chi^{\prime}, \theta^{\prime}\right)$ with $\chi+\theta=\chi^{\prime}+\theta^{\prime}$, obtained by adding a copy of $\theta_{j_{0}}$ every $q$ sections (so not to destroy the $q$-periodicity):

$$
\begin{aligned}
& \theta^{\prime}=\theta+\sum_{n \in D}\left(\theta_{j_{0}}+n q e_{k}\right), \\
& \chi^{\prime}=\chi+\sum_{n \in D}\left(\theta_{j_{0}}+n q e_{k}\right),
\end{aligned}
$$

where $D$ is the set of integers $n$ such that $j_{0}+n q \in\left[0, N_{k}\right]$, and $e_{k}$ is the unit vector in the direction of the $k^{\text {th }}$ coordinate axis. Certainly $\chi^{\prime} \in L_{k}(\Lambda)$ and $\theta^{\prime} \in L_{\leqq k-1}(\Lambda)$ and moreover:

$$
\left|\chi^{\prime}\right|=|\chi|+\sum_{n \in D}\left[\left|\theta_{j_{0}}\right|-2\left|\chi \cup \theta_{j_{0}}\right|\right]
$$

Hence, by (4.33)

$$
\left|\chi^{\prime}\right|<|\chi|
$$

against the assumed economicity of the original decomposition $(\chi, \theta)$. This contradiction proves (4.32), hence the lemma and with it the inequality (4.23).

The combination of Proposition 4.5 with (4.18) and Proposition 4.3 yields the uniqueness result of Theorem 3.2. 


\section{More General Abelian Groups}

The results summarized in Sect. 3.2 generalize to systems where the spin at each site takes values in the group $\mathbf{Z}_{\mathrm{p}}=\{0,1, \ldots, p-1\}$ of integers modulo $p$. Theorem 3.2 requires the restriction that $p$ be a prime. In this section we sketch the proofs of these extensions.

\subsection{Definitions and Notation}

As for spin- $\frac{1}{2}$, the configuration space

$$
\mathbf{X}=\mathbf{Z}_{p}^{\mathbf{Z}^{v}}
$$

is endowed with the product group structure and topology. The characters of $\mathbf{X}$ are functions of the form

$$
\sigma_{B}(X)=\prod_{a} e^{2 \pi i B(a) X(a) / p}=\prod_{a} \sigma_{a}^{B(a)},
$$

where $B$-a multiplicity function - is an element of $\mathbf{Z}_{\mathrm{p}}^{\left(\mathbf{Z}^{v)}\right.}$, i.e., a $\mathbf{Z}_{\mathrm{p}}$-valued function which is zero except on a finite set of points of $\mathbf{Z}^{v}$, called support of $B$ and denoted $\operatorname{supp} B$. For $p=2(\operatorname{spin} 1 / 2)$ we recover the notions of Sect. 3. The set of characters forms a group denoted $\mathbf{X}^{*}$, which is isomorphic to the group of multiplicity functions with coordinatewise sum modulo $p$. The latter is in fact a ring with product

$$
A \cdot B(x)=\sum_{y+z=x} A(y) B(z)
$$

which is also written as

$$
A \cdot B=\sum_{x} A(x) B+x
$$

which is a generalization of (2.6). Here " $\sum$ " is the coordinatewise sum modulo $p$, $A(x) B$ is defined by $(A(x) B)(y)=A(x) B(y)(\bmod p)$, and for $C \in \mathbf{Z}_{\mathrm{p}}^{\left(\mathbf{Z}^{\nu}\right)}$ and $x \in \mathbf{Z}^{v}, C+x$ denotes the translated $(C+x)(y)=C(x-y)$. The ring so obtained, in fact an algebra over $\mathbf{Z}_{\mathrm{p}}$, is the standard group algebra $\mathbf{Z}_{\mathrm{p}}\left[\mathbf{Z}^{v}\right]$ of $\mathbf{Z}^{v}$ with coefficients in $\mathbf{Z}_{\mathrm{p}}$.

The Hamiltonians are functions of the form

$$
H=-\sum_{B \in \mathscr{B}} J(B) \sigma_{B},
$$

where $\mathscr{B}$ is a $\mathbf{Z}^{v}$-invariant family of multiplicity functions, and the complex numbers $\{J(B)\}$ satisfy $J(-B)=\overline{J(B)}$ so that Hamiltonians are real-valued. The range of the interaction is the supremum of the diameters of the supports of the multiplicity functions in $\mathscr{B}$. The interaction is real if $J(B)$ is real for each $B$, and ferromagnetic if $J(B)>0$ for each $B$. The Gibbs state $\varrho^{+}$is defined by placing as boundary condition the function $\mathbf{1}$, where $\mathbf{1}_{a}=0_{\mathbf{Z}_{\mathrm{p}}}$ for each $a \in \mathbf{Z}^{v}$.

All the results summarized in Sect. 3.1 have natural generalizations to the present setting. The ring structure of the set of bonds is now that of $\mathbf{Z}_{\mathrm{p}}\left[\mathbf{Z}^{v}\right]$, which is also a unique factorization domain if $p$ is prime. Results $(2.8)$ and $(2.5)$ hold, $\overline{\mathscr{B}}$ being the ideal of $\mathbf{Z}_{\mathrm{p}}\left[\mathbf{Z}^{\nu}\right]$ generated by $\mathscr{B}$. 


\subsection{Proof of Theorem 3.1 for a General Finite Abelian Group at Each Lattice Site}

We discuss here only the points where the proof differs from that of Sect.3.3.

We first note that even when the characters (5.1) are complex-valued, for ferromagnetic Hamiltonians the correlations $\varrho^{+}\left(\sigma_{B}\right)$ are real (Bochner's theorem), in fact nonnegative $[18,28]$. Proceeding as in Sect. 3.3, we see that we must show

$$
\sum_{X, Y \in \mathbf{X}_{A}}\left[\operatorname{Re} \sigma_{A}(X)-\operatorname{Re} \sigma_{A}(Y)\right] e^{-L_{2}(X)-L_{1}(Y)} \geqq 0 .
$$

We then observe that to prove this is enough to show that

$$
e^{-L_{2}(X)+L_{1}(X)} \text {. }
$$

is positive definite for every $\Lambda \in \mathscr{P}_{f}\left(\mathbf{Z}^{v}\right)$. Indeed, if this is true, then there are numbers $t_{C}>0$ such that

$$
e^{-L_{2}(X)+L_{1}(X)}=\sum_{\sigma_{C} \in \mathbf{X}_{A}^{*}} t_{C} \operatorname{Re} \sigma_{C}(X),
$$

and hence

$$
\begin{aligned}
\text { LHS of }(5.3)= & \sum_{X, Y \in \mathbf{X}_{A}}\left[\operatorname{Re} \sigma_{A}(X)-\operatorname{Re} \sigma_{A}(Y)\right] e^{-L_{2}(X)+L_{1}(X)} e^{-L_{1}(X)-L_{1}(Y)} \\
= & \sum_{\sigma_{C} \in \mathbf{X}_{A}^{*}} t_{C} \sum_{X, Y \in \mathbf{X}_{A}} \operatorname{Re} \sigma_{C}\left[\operatorname{Re} \sigma_{A}(X)-\operatorname{Re} \sigma_{A}(Y)\right] e^{-L_{1}(X)-L_{1}(Y)} \\
= & \sum_{\sigma_{C} \in \mathbf{X}_{A}^{*}} t_{C} \frac{1}{2} \sum_{X, Y \in \mathbf{X}_{A}}\left[\operatorname{Re} \sigma_{C}(X)-\operatorname{Re} \sigma_{C}(Y)\right] \\
& \times\left[\operatorname{Re} \sigma_{A}(X)-\operatorname{Re} \sigma_{A}(Y)\right] e^{-L_{1}(X)-L_{1}(Y)} \\
\geqq & 0 .
\end{aligned}
$$

The last inequality is a well known result (see e.g. [18, Example 4]).

The positive definiteness of (5.4) is a particular case of the following lemma.

Lemma 5.1. Let $\mathscr{G}$ be a finite Abelian group; $f, g$ real valued functions on $\mathscr{G}$ such that

i) $f$ is positive definite,

ii) the Fourier transform $\hat{g}$ of $g$ is real-valued, and

iii) for any $\sigma \in \mathscr{G}^{*}$,

$$
\widehat{f^{n}}(\sigma)=0 \quad \text { for all } n \Rightarrow \hat{g}(\sigma)=0
$$

Then the function

$$
e^{s f+g}
$$

is positive definite for $s \in \mathbf{R}$ large enough.

Proof. We could proceed as in the spin- $\frac{1}{2}$ case using the HTE. However we prefer to deduce the lemma from a corresponding result on positive definite matrices, which is of independent interest. 
First, in a standard way, by considering convolution operators, we let to any function $f$ on $\mathscr{G}$ correspond a $\mathscr{G}^{*} \times \mathscr{G}^{*}$ matrix $F$ :

$$
F_{\sigma \tau}=\hat{f}\left(\sigma \tau^{-1}\right) \text {. }
$$

The relation

$$
\widehat{f g}\left(\sigma \tau^{-1}\right)=\sum_{\gamma \in \mathscr{G} *} \hat{f}\left(\sigma \gamma^{-1}\right) \hat{g}\left(\gamma \tau^{-1}\right)
$$

proves that (5.7) is an algebra isomorphism between the real-valued functions of $\mathscr{G}$ and a commutative algebra of matrices with indices on $\mathscr{G}^{*}$. It is simple to check that the isomorphism is such that:

$$
f \text { is real } \Leftrightarrow F \text { is Hermitian }
$$

and

$f$ is positive definite $\Leftrightarrow F$ has non-negative entries.

Moreover, hypothesis (5.6) is equivalent to the property

$$
\left(F^{n}\right)_{\sigma, \tau}=0 \forall n \Rightarrow(G)_{\sigma, \tau}=0 \text {. }
$$

Therefore we see that the lemma is a consequence of the following claim.

Claim. Let $F, G$ be real symmetric commuting matrices. In addition assume that $F$ has non-negative entries and that the matrices satisfy (5.8). Then the matrix

$$
e^{s F+G}
$$

has non-negative entries for $s$ large enough.

Proof of the Claim. ${ }^{1}$ Property (5.8) allows us to assume without loss of generality that for each $\sigma, \tau \in \mathscr{G}^{*}$ there exists some $n$ for which

$$
\left(F^{n}\right)_{\sigma, \tau}>0 \text {. }
$$

Indeed, otherwise we could classify the indices into groups $I_{k}$ such that $\left(F^{n}\right)_{\sigma, \tau}=0$ for every $n$ when $\sigma$ and $\tau$ belong to different $I_{k}$. This classification is well defined because $F$ is symmetric. By (5.8) also $G_{\sigma, \tau}=0$ when $\sigma$ and $\tau$ belong to different $I_{k}$. Therefore $F$ decompose into block matrices along the diagonal for which (5.10) is true, and it is enough to study (5.9) for each such block.

The inequality (5.10) is exactly what we need to be able to apply PerronFrobenius Theorem to the matrix $F$. As a consequence, of all the eigenvalues $\lambda_{k}$ of $F$ there is one of them, $\lambda_{0}$, such that

$$
\left|\lambda_{k}\right|<\lambda_{0}, \quad k \neq 0
$$

The eigenvalue $\lambda_{0}$ has multiplicity one, and the components $\left(v_{0} \mid e_{i}\right)$ of the corresponding eigenvector $v_{0}$ with respect to the canonical basis $e_{i}$ are strictly positive:

$$
\left(v_{0} \mid e_{i}\right)>0 \quad i=1, \ldots\left|\mathscr{G}^{*}\right|
$$

\footnotetext{
${ }^{1}$ We owe this proof to Michael Aizenman
} 
Moreover, $F$ and $G$ can be diagonalized simultaneously because they commute (and are symmetric). If $\left\{v_{k}\right\}$ is the set of simultaneous eigenvectors and $\gamma_{k}$ the $G$-eigenvalues; we have

$$
\begin{aligned}
\left(e_{i} \mid e^{s F+G} e_{j}\right) & =\sum_{k}\left(e_{i} \mid v_{k}\right)\left(v_{k} \mid e^{s F+G} e_{j}\right) \\
& =\sum_{k, j}\left(e_{i} \mid v_{k}\right)\left(v_{k} \mid e_{j}\right) e^{s \lambda_{k}+\gamma_{k}} \\
& =\left(e_{i} \mid v_{0}\right)\left(v_{0} \mid e_{j}\right) e^{s \lambda_{0}+\gamma_{0}}+\sum_{j} \sum_{k \neq 0}\left(e_{i} \mid v_{k}\right)\left(v_{k} \mid e_{j}\right) e^{s \lambda_{k}+\gamma_{k}}
\end{aligned}
$$

By (5.11) and (5.12) the first summand in (5.13) dominates for large $s$. Therefore

$$
\left(e_{i} \mid e^{s F+G} e_{j}\right) \geqq 0
$$

for large s. This proves the claim, and hence Lemma (5.1) and Theorem 3.1. Combining (5.13) with the requirement (5.14) one could obtain an explicit relation for $s$ of the type (3.20).

\subsection{Generalization of Theorem 3.2}

for an Abelian Group of Prime Order at Each Lattice Site

A system is trivial if $\mathscr{B}$ is formed by translating multiples of a single multiplicity function $C$, called fundamental bond:

$$
\mathscr{B}_{C}=\left\{k C+x: k \in \mathbf{Z}_{\mathrm{p}}, x \in \mathbf{Z}^{v}\right\} .
$$

A system is factorizable if every multiplicity function of $\mathscr{B}$ is the tensor product of one-dimensional functions. In particular a factorizable trivial system has a fundamental bond of the form

$$
C(x)=C_{1}\left(x_{1}\right) \ldots C_{v}\left(x_{v}\right),
$$

where $C_{i}: \mathbf{Z} \rightarrow \mathbf{Z}_{\mathrm{p}}, 1 \leqq 1 \leqq v$. Equivalently, $C=C_{1} \cdot \ldots \cdot C_{v}$ with "." defined in (5.2).

Theorem 5.2. Let $p$ be prime, and consider the factorizable trivial system defined by

$$
H=-\sum_{B \in \mathscr{B}_{C}} J(B) \sigma_{B}
$$

such that

$$
|J(B)| \leqq J \leqq \infty
$$

Let $r$ be a positive number and let the interaction $K=-\sum J_{3}(B) \sigma_{B}$ have range smaller than $r$. Then, there exists a number $R_{r, C}(J)$ such that if

$$
\sup _{B}\left|J_{3}(B)\right|<R_{r, C}(J),
$$

the set of Gibbs states $\Delta(H+K)$ has exactly one element.

Sketch of the Proof. Basically, we only need to discuss how to define the reduced partition functions in the present more general setting. The proof is then a straightforward generalization of that for spin $\frac{1}{2}$. 
The HTE for a trivial system takes the following form [21]. We introduce the potentials $H_{(x)}$ obtained by summing all the terms of (5.15) corresponding to multiples of the same bond, i.e., by writing (5.15) as

$$
H=-\sum_{x \in \mathbf{Z}^{v}} \sum_{k \in \mathbf{Z}_{\mathrm{p}}} J(k C+x) \sigma_{k C+x}=\sum_{x \in \mathbf{Z}^{v}} H_{(x)} .
$$

We also identify families of bonds with subsets of $\mathbf{Z}^{v} \times \mathbf{Z}_{\mathrm{p}}$ [cf. (4.19)]

$$
\alpha \subset \mathscr{B} \leftrightarrow\{(x, k): k C+x \in \alpha\} .
$$

In particular, the family of bonds of the Hamiltonian $H_{\Lambda}(\cdot \mid Y)$, with boundary condition $Y \in X_{M}, M \subset \Lambda^{c}$, is identified with $\mathscr{B}(\Lambda \mid Y)=\{(x, k): \operatorname{supp}(k C+x)$ intersects $\Lambda$ and $\Lambda \cup M\}$; and the corresponding set of cycles is identified with

$$
\mathscr{K}(\Lambda \mid Y)=\left\{\alpha \subset \mathscr{B}(\Lambda \mid Y):\left[\sum_{(x, k) \in \alpha}(k C+x)\right] \cap \Lambda=0\right\} .
$$

We remark that both $\mathscr{B}(\Lambda \mid Y)$ and $\mathscr{K}(\Lambda \mid Y)$ depend on $Y$ only through $M$, and moreover $\mathscr{K}(\Lambda \mid Y) \subset \mathscr{K}(\Lambda \mid \mathbf{1}) \equiv \mathscr{K}_{\Lambda}$.

The HTE of the partition function $Z(\Lambda \mid Y)$ is obtained by expanding each factor $e^{-H_{(x)}}$ in Fourier series and taking the trace. The result is

$$
Z(\Lambda \mid Y)=\left[\prod_{x} \sum_{X} e^{-H_{(x)}(X \mid Y)}\right] Z^{0}(\Lambda \mid Y)
$$

with

$$
Z^{0}(\Lambda \mid Y)=\sum_{\alpha \in \mathscr{K}(\Lambda \mid Y)} \prod_{(x, k) \in \alpha} t(x, k \mid Y)
$$

where

$$
t(x, k \mid Y)=\frac{\sum_{X} \sigma_{k C+x}(X) e^{-H_{(x)}(X \mid Y)}}{\sum_{X} e^{-H_{(x)}(X \mid Y)}} .
$$

It is simple to check that the bound (4.16) remains valid in the present setting, and hence so does Proposition 4.3. Moreover, by a Griffiths inequality [28]:

$$
|t(x, k \mid Y)| \leqq t_{|J|}\left(x, k \mid \mathbf{1}_{M}\right),
$$

where $t_{|J|}$ corresponds to the interaction in which all the $J(k C+x)$ are replaced by the upper bound $|J|$ and $Y$ is replaced by $\mathbf{1}_{M}$. Also, $t_{|J|}\left(x, k \mid \mathbf{1}_{M}\right)<1$ for each $(x, k) \in \mathscr{B}(\Lambda \mid \mathbf{1}) \equiv \Lambda^{*}$. Therefore

$$
\left|Z^{0}(\Lambda \mid Y)-1\right| \leqq \sum_{\substack{\alpha \in \mathscr{K}_{\Lambda} \\ \alpha \neq 0}} \tau(J)^{\mid \text {supp } \alpha \mid} .
$$

With $\tau(J)=\max \left\{t_{|J|}(x, k \mid \mathbf{1}):(x, k) \in \Lambda^{*}\right\}<1$. It is shown in the appendix that Proposition 4.4 is valid as long as $p$ is prime (this is the only part of the proof of Theorem 5.2 where the prime character of $p$ is used), using " $\sum$ " as the sum modulo $p$ and $\Lambda^{*}=\mathscr{B}(\Lambda \mid \mathbf{1})$. The proof of Proposition 4.5 can then be repeated replacing throughout the symmetric difference by the sum modulo $p$. 


\subsection{Comparison with Other Works and Concluding Coments}

Our work on many phase transitions started with Monte-Carlo stimulations of the Benchmark Model. The present construction was essentially completed in the spin- $\frac{1}{2}$ case during the Summer of 1982, before we became aware of [28] and, more recently, of $[1,2]$. Theorem 3.2 was proved at first in a more involved way using the Dobrushin-Pechersky uniqueness theorem [7] (cf. [13]). Since the work [28] appeared so much earlier, comparison of the present construction with that of Pfister is in order.

In both constructions, to show that certain order parameters are zero one compares the original model with another one, using correlation inequalities. In [28] the comparison model, about which one has to have an independent information, is either defined on a reduced lattice, as in the case of the AshkinTeller model, or has a reduced configuration space. Thus to define models with more and more phase transitions one has to consider larger and larger configuration spaces. The comparison of the models is done with a help of an inequality by Ginibre.

In our case Theorem 3.1 yields a comparison with a model on the same lattice, so that one can construct spin- $\frac{1}{2}$ models with many phase transitions on the simple lattice. Vanishing of correlations in the comparison model is obtained from the uniqueness Theorem 3.2. We do not know how one could use Pfister's method to obtain our results.

To further elucidate these comments we discuss an example of $[1,2]$ and [28] using our method. Let $n=k \cdot m, k, m \neq 1$, and consider the system with configuration space $\mathbf{Z}_{n}^{\mathbf{Z}^{v}}$ and Hamiltonian

$$
H=-\mu \sum_{n . n .} \sigma_{a} \bar{\sigma}_{b}-\sum_{n . n .} \sigma_{a}^{k} \bar{\sigma}_{b}^{k},
$$

where

$$
\sigma_{a}(X)=\exp 2 \pi i X_{a} / n .
$$

(The case of the circle is reduced to the one considered here using Lemma 5.2 of [28], as in [28]; see also [11]). One wants to show that for $\mu$ small enough there are at least two changes in $\mathscr{A}_{\beta}$ : at low temperatures $\left\langle\sigma_{0}\right\rangle^{+} \neq 0$, at high temperatures both $\left\langle\sigma_{0}\right\rangle^{+}=0$ and $\left\langle\sigma_{0}^{k}\right\rangle^{+}=0$, whereas at intermediate temperatures $\left\langle\sigma_{0}\right\rangle^{+}=0$ while $\left\langle\sigma_{0}^{k}\right\rangle^{+} \neq 0$. The only nontrivial point is the proof of the last statement.

We first choose a $\beta^{\prime}$ so large that for $\mu=0$

$$
\left\langle\sigma_{0}^{k}\right\rangle_{\beta H}^{+} \neq 0 \text { for } \beta>\beta^{\prime} .
$$

By GKS (5.2) holds for $\mu>0$ too. Then applying Theorem 3.1 to $H_{1}=-\sum_{n . n .} \sigma_{a}^{k} \bar{\sigma}_{b}^{k}$, $H_{2}=-\sum_{a}\left(\sigma_{a}^{k}+\bar{\sigma}_{a}^{k}\right)$ and $K=-\beta \mu \sum_{n . n .} \sigma_{a} \bar{\sigma}_{b}$, we obtain the majorization

$$
\left\langle\sigma_{0}\right\rangle_{\beta H_{1}+K}^{+} \leqq\left\langle\sigma_{0}\right\rangle_{f(\beta) H_{2}+K}^{+}
$$

Now, $\mathrm{H}_{2}$ has "one-point" interaction only. Therefore the DobrushinShlosman Theorem 4.1 applies to it trivially: $\varrho_{\Lambda}(\cdot \mid Y)$ is independent of $Y$ and the right-hand side of (4.6) is zero. Hence small perturbations of $f(\beta) \mathrm{H}_{2}$ have unique 
Gibbs states, and since $H_{2}$ and $K$ are invariant under shifting all the spins by $m$, we conclude that $\left\langle\sigma_{0}\right\rangle_{f(\beta) H_{2}+K}^{+}$is zero for $\beta$ close to $\beta^{\prime}$ and $\mu$ small enough. This concludes the proof. Choosing $n=k_{1} \cdot \ldots \cdot k_{r}$ one can define systems with $r$ phase transitions.

One could try to combine our construction with Pfister's to describe the situation for general $\mathbf{Z}_{\mathrm{n}}$-models, with composite $n$. The case when $n$ is a product of distinct primes should present no new problems since for this case one has a description of $\mathscr{A}_{\infty}$ through a greatest common divisor, as when $n$ is prime [26]. However, while for the Hamiltonian (5.1) the choice of the comparison Hamiltonian $\mathrm{H}_{2}$ was obvious, and $\mathrm{H}_{2}$ was easy to analyse, the description of $\mathscr{A}_{\infty}$ through generators has not been worked out in the general case, although one does have a reduction process for general $n$ (and general lattices). Though we consider it likely that $\mathscr{A}_{\infty}$ should in general have a set of generators which define a model for which an analogue of Theorem 3.2 is true; even with this problem solved, the problem of determination of most general chains $\ldots \mathscr{C}_{n-1} \subset \mathscr{A}_{n} \subset \mathscr{A}_{n+1} \subset \ldots$ of order parameters would still be open.

\section{A. Cycles for Factorizable Systems}

We present here the proof of Proposition 4.4 for the general case of a discrete set of prime cardinality at each lattice site. The proof relies on the properties of the algebraic structure of the configuration space $\mathbf{X}=\mathbf{Z}_{\mathrm{p}}^{\mathbf{Z}^{\nu}}$ and its dual $\hat{\mathbf{X}}=\mathbf{Z}_{\mathrm{p}}^{\left(\mathbf{Z}^{\nu}\right)}$. Both $\mathbf{X}$ and $\hat{\mathbf{X}}$ have a canonical (product) group structure and, moreover, $\hat{\mathbf{X}}$ is an algebra -the group algebra $\mathbf{Z}_{\mathrm{p}}\left[\mathbf{Z}^{v}\right]$ of $\mathbf{Z}^{v}$ with coefficients in $\mathbf{Z}_{\mathrm{p}}$, with the product defined by

$$
(A \cdot B)(y)=\sum_{x} A(x) B(y-x) .
$$

If $p$ is prime, $\mathbf{Z}_{\mathrm{p}}$ is a field and $\mathbf{Z}_{\mathrm{p}}\left[\mathbf{Z}^{v}\right]$ is a unique factorization domain. In the sequel we shall adapt our notation to the simpler case of a trivial system by resorting to the identification (5.17). For a fixed multiplicity function $C$ (which can be thought of as the fundamental bond of a trivial system). We shall denote

$$
S_{C}=\left\{X \in \mathbf{X}: \sigma_{C+a}(X)=1 \text { for every } a \in \mathbf{Z}^{\nu}\right\}
$$

(symmetry group for $C$ ),

$$
\mathscr{K}^{C}=\left\{\alpha \in \mathbf{Z}_{\mathrm{p}}^{\mathbf{Z}^{v}}: \prod_{x} \sigma_{\alpha(x) C+x}=1\right\} .
$$

The elements of $\mathscr{K}^{C}$ are called infinite cycles for (the trivial system with fundamental bond) $C$. Note that the product makes sense because $C$ has finite support. The annihilator of $S_{C}$ :

$$
S_{C}^{\perp}=\left\{A \in \mathbf{Z}_{\mathrm{p}}^{\mathbf{Z}^{v}}: \sigma_{A}(X)=1 \forall X \in S_{C}\right\}
$$

is known to be generated by the set

$$
\mathscr{B}_{f}(C)=\left\{A \cdot C: A \in \mathbf{Z}_{\mathrm{p}}\left[\mathbf{Z}^{v}\right]\right\} .
$$

Let us also introduce the involution $I: \mathbf{Z}_{\mathrm{p}}\left[\mathbf{Z}^{v}\right] \rightarrow \mathbf{Z}_{\mathrm{p}}\left[\mathbf{Z}^{v}\right]$ defined by

$$
I(B)(x)=B(-x) \text {. }
$$


We then have the following relation between infinite cycles and symmetry groups:

$$
\alpha \in \mathscr{K}^{C} \Leftrightarrow \sum_{B \in \alpha} B \in S_{I(C)},
$$

which is a consequence of the identity

$$
\left[\prod_{x \in \mathbf{Z}^{v}} \sigma_{\alpha(x) C+x}\right]_{s}\left(1_{\mathbf{z}_{\mathbf{p}}}\right)=\sigma_{I(C)+s}\left(\sum_{B \in \alpha} B\right)
$$

valid for every $s \in \mathbf{Z}^{v}$.

Therefore, to characterize the cycles of trivial systems it is equivalent to study the symmetry group of the inverted of the fundamental bond. This task can be further decomposed when the fundamental bond can be factored into coprime factors.

Proposition A.1. If $C, C_{1}, C_{2} \in \mathbf{Z}_{\mathrm{p}}\left[\mathbf{Z}^{v}\right]$-p prime-are such that $C=C_{1} \cdot C_{2}$ with $C_{1}$ and $C_{2}$ coprime; then $S_{C}$ is generated by $S_{C_{1}}$ and $S_{C_{2}}$.

Proof. Let us denote $\widetilde{S}$ the subgroup generated by $S_{C_{1}}$ and $S_{C_{2}}$. Then $\widetilde{S}^{\perp}$ is generated by $\mathscr{B}_{f}\left(C_{1}\right) \cap \mathscr{B}_{f}\left(C_{2}\right) \subset \mathscr{B}_{f}(C)$, which implies $S_{C}^{\perp} \subset \widetilde{S}^{\perp}$. Indeed, if $A \in \mathscr{B}_{f}\left(C_{1}\right)$ $\cap \mathscr{B}_{f}\left(C_{2}\right)$ there exist multiplicity functions $Q(1), Q(2) \in \mathbf{Z}_{\mathrm{p}}\left[\mathbf{Z}^{\nu}\right]$ such that

$$
Q(1) \cdot C_{1}=A=Q(2) \cdot C_{2} \text {. }
$$

As $\mathbf{X}^{*}$ is a unique factorization domain and $C_{1}$ and $C_{2}$ are coprime, this implies

$$
A=Q \cdot C_{1} \cdot C_{2}=Q \cdot C
$$

for some $Q \in \mathbf{Z}_{\mathrm{p}}\left[\mathbf{Z}^{v}\right]$. Hence $A \in \mathscr{B}_{f}(C)$.

A factorizable trivial system is defined by a fundamental bond of the form

$$
C=C_{1} \cdot \ldots \cdot C_{v},
$$

where each $C_{i}$ has support contained in the $i^{\text {th }}$ coordinate axis. Then $I(C)=I\left(C_{1}\right) \cdot \ldots \cdot I\left(C_{v}\right)$ is also the (ring) product of multiplicity functions with supports along each axis. By (A.2) and Proposition A.1, the properties of the cycles of such system can be deduced from the study of one-dimensional trivial systems.

Proposition A.2. Consider a trivial system with configuration space $\mathbf{Z}_{\mathrm{p}}^{\mathbf{Z}}$, p prime, and fundamental bond $C$. Then, if $|\operatorname{supp}(C)|=m$ :

i) If $X, Y \in S_{C}$ agree on a set of $m-1$ consecutive points, then $X=Y$.

ii) $\left|S_{C}\right|=p^{m-1}$.

iii) There exists a positive integer $q$ such that every $X \in S_{C}$ has period $q$.

Proof. i) Let $T$ be the subset of $\mathbf{Z}$ where $X$ and $Y$ agree. We claim that $\max (T)=\infty$. Indeed, if $\max (T)=b<\infty$, then we can consider the bond $C+b-m+1$ with support in $[b-m+1, b+1]$. For $p$ prime it is easy to see that the condition $1=\sigma_{C+b-m+1}(X)=\sigma_{C+b-m+1}(Y)$ implies $X_{b+1}=Y_{b+1}$ against the maximality of $b$. The proof that $\min (T)=-\infty$ is analogous.

ii) By i) $\left|S_{C}\right| \leqq p^{m-1}$. The opposite inequality follows from the fact that every configuration on $[0, \ldots, m-2]$ can be uniquely extended to an element of $S_{C}$. 
iii) By ii) each $X \in S_{C}$ has period smaller than $p^{m-1}$. Choose $q$ as the lowest common multiple of the periods of the $p^{m-1}$ configurations of $S_{C}$.

Finally, we obtain the desired characterization. For a finite set $\Lambda \in \mathbf{Z}^{v}$, let $\Lambda^{*}=\left\{x \in \mathbf{Z}^{v}: \operatorname{supp}(C+x) \cap \Lambda \neq \emptyset\right\}$, and

$$
\mathscr{K}_{\Lambda}=\left\{\alpha \in \mathbf{Z}_{\mathrm{p}}^{\mathbf{Z}^{v}}: \operatorname{supp}(\alpha) \in \Lambda^{*}, \operatorname{supp}\left(\sum_{x} \alpha(x) C+x\right) \cap \Lambda=\emptyset\right\} .
$$

Theorem A.3. For a factorizable trivial system with single spin space $\mathbf{Z}_{\mathrm{p}}$, p prime, there exists an integer $q$ such that for each parallelepiped $A$

$$
\alpha \in \mathscr{K}_{\Lambda} \Rightarrow \alpha=\sum_{i=1}^{v} \alpha(i)
$$

with $\operatorname{supp}(\alpha) \subset \Lambda^{*}$ and each $\left.\alpha(i)\right|_{\Lambda^{*}}$ periodic in the $i^{\text {th }}$ direction.

Proof. For $\Lambda=\mathbf{Z}^{v}$ the result is just a combination of the previous three propositions. For $A$ with some side of finite size, the result follows from the fact that each $\alpha \in \mathscr{K}_{\Lambda}$ can be extended to an infinite cycle.

Acknowledgements. J. S. would like to thank Israel Sigal for warm hospitality at the Mathematics Department of the Weizmann Institute of Science during the Summer of 1982, where most of his contribution to this paper was worked out. His work has been supported in part by NSF Grant DMS-8700580.

\section{References}

1. Alcaraz, F.C., Koberle, R.: Duality and the phases of $Z(N)$ spin systems. J. Phys. A 13, L153 (1980)

2. Alcaraz, F.C., Koberle, R.: The phases of two-dimensional spin and four-dimensional gauge systems with $Z(N)$ symmetry. J. Phys. A 14, 1169 (1981)

3. Bricmont, J., Kuroda, K., Lebowitz, J.L.: First order phase transitions in lattice and continuous systems: extension of the Pirogov-Sinai theory. Commun. Math. Phys. 101, 501 (1985)

4. Bricmont, J., Slawny, J.: First order phase transitions and perturbation theory. In: Statistical mechanics and field theory: mathematical aspects. Hugenholtz, N.M., Winnink, M. (eds.). Berlin, Heidelberg, New York: Springer 1986

5. Briemont, J., Slawny, J.: Phase transitions with a finite number of dominant ground states. To appear in J. Stat. Phys.

6. Dobrushin, R.L.: The existence of a phase transition in the two- and three-dimensional Ising models. Teorija Verojatn. Prim. 10, 209 (1965)

7. Dobrushin, R.L., Pecherski, E.A.: Uniqueness conditions for finitely dependent random fields. In: Colloquia mathematica societatis Janos bolyai, Vol. 27. Random Fields, Hungary 1979

8. Dobrushin, R.L., Shlosman, S.B.: Constructive criterion for the uniqueness of Gibbs fields. Statistical physics and dynamical systems, pp.347-370. Fritz, J., Jaffe, A., Szasz, D. (eds.). Boston, Basel, Stuttgart: Birkhäuser 1985

9. Dobrushin, R.L., Zahradnik, M.: Phase diagrams for the continuous spin models. Extension of Pirogov-Sinai theory. In: Mathematical problems of statistical physics and dynamical problems. Dobrushin, R.L. (ed.). Dordrecht: Reidel

10. Dudley, R.M.: Probabilities and metrics. Aarhus University, Lecture Notes, Vol. 45, 1976

11. Elitzur, E., Pearson, R.B., Shigemitsu, J.: Phase structure of discrete abelian spin and gauge systems. Phys. Rev. D 19, 3698 (1979) 
12. Fisher, M.E.: Critical temperatures of anisotropic Ising lattices. II. General upper bounds. Phys. Rev. 162, 480-485 (1967)

13. Fernández, R.: Study of ferromagnetic systems with many phase transitions. Thesis, Virginia Polytechnic Institute and State University (1984)

14. Fröhlich, J., Simon, B., Spencer, T.: Infrared bounds, phase transitions and continuous symmetry breaking. Commun. Math. Phys. 50, 79 (1976)

15. Fröhlich, J., Israel, R., Lieb, E.H., Simon, B.: Phase transitions and reflection positivity. I. General theory and long range lattice models. Commun. Math. Phys. 62, 1 (1978)

16. Fröhlich, J., Israel, R., Lieb, E.H., Simon, B.: J. Stat. Phys. 22, 297 (1980)

17. Ginibre, J.: Correlations in Ising ferromagnets. Cargèse Lectures in Physics, Vol. 4, Kastler, D. (ed.). New York, London, Paris: Gordon and Breach

18. Ginibre, J.: General formulation of Griffith's inequalities. Commun. Math. Phys. 16, 310 (1970)

19. Griffiths, R.B.: Peierls proof of spontaneous magnetization in a two-dimensional Ising ferromagnet. Phys. Rev. 136A, 437 (1964)

20. Griffiths, R.B.: Correlations in Ising ferromagnets. I. J. Math. Phys. 8, 478 (1967)

21. Gruber, C., Hinterman, A., Merlini, D.: Group analysis of classical lattice systems. Lecture Notes in Physics, Vol. 60. Berlin, Heidelberg, New York: Springer 1977

22. Holsztynski, W.: Communique at Yeshiva University Meeting on Statistical Mechanics, December 1976

23. Holsztynski, W., Slawny, J.: Phase transitions in ferromagnetic spin systems at low temperatures. Commun. Math. Phys. 66, 147 (1979)

24. Kelly, D.G., Sherman, S.: General Griffith's inequalities on correlations in Ising ferromagnets. J. Math. Phys. 9, 466 (1968)

25. Kotecky, R., Preiss, D.: An inductive approach to Pirogov-Sinai theory. Proc. Winter School on Abstract Analysis 1983. [Suppl.] Ai. Rend del Circ. Mat. di Palermo (1983)

26. Miekisz, J.: The decomposition property and equilibrium states of ferromagnetic lattice systems. Commun. Math. Phys. 109, 353 (1987)

27. Peierls, R.: On Ising's model ferromagnetism. Proc. Camb. Phil. Soc. 32, 477 (1936)

28. Pfister, C.-E.: Translation invariant equilibrium states of ferromagnetic abelian lattice systems. Commun. Math. Phys. 86, 375 (1982)

29. Pirogov, S.A., Sinai, Ya.G.: Phase diagrams of classical lattice systems. I, II. Theor. Math. Phys. 25, 1185 (1976); 26, 39

30. Slawny, J.: A family of equilibrium states. Commun. Math. Phys. 35, 297 (1974)

31. Slawny, J.: Ferromagnetic systems with local symmetries (unpublished) (1979)

32. Slawny, J.: Low temperature properties of classical lattice systems: Phase transitions and phase diagrams. In: Phase transitions and critical phenomena, Vol. 11. Domb, C., Lebowitz, J.L. (eds.). London: Academic Press 1987

33. Wegner, F.: Duality in generalized Ising models and phase transitions without local order parameters. J. Math. Phys. 12, 2259 (1971)

34. Zahradnik, M.: Low temperature continuous spin Gibbs states on a lattice and the interfaces between them - A Pirogov-Sinai type approach. To appear in Proc. Intern. Conf. "Statistical Mechanics and Field Theory; Mathematical Aspects," Hugenholtz, N.M., Winnik, M. (eds.). Groningen, Aug. 1985

35. Zahradnik, M.: An alternate version of Pirogov-Sinai theory. Commun. Math. Phys. 93, 559 (1984)

Communicated by M. Aizenman

Received January 20, 1988; in revised form August 10, 1988 